\title{
Ist für einen Fabrikbetrieb der Anschluß an ein Elektrizitätswerk oder eine eigene Kraftanlage vorzuziehen?
}

\author{
Von \\ Dr. Walter Straus, Charlottenburg.
}

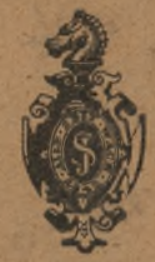

Berlin.

Verlag von Jutius Springer

1914.

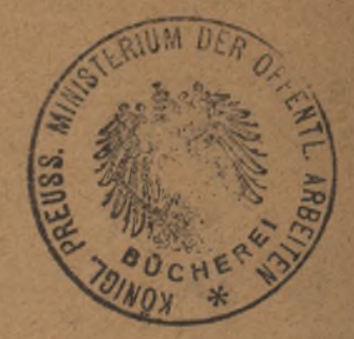

${ }^{B \cdot 3^{l}}$ is

71.14 .169 
Biblioteka Politechniki Krakowskiej 


\title{
Sonderabdruck aus der \\ ELEKTROTECHNISCHEN ZEITSCHRIFT \\ 1914. Heft 21 und 22.
}

Nachdruck nur mit Genehmigung des Verfassers und der Redalktion der Elektrotechnischen Zeitschrift und mit Quellenangabe gestattet.)

\section{BIBLIOTERA PCLITECHNIGZNA KRA KO W III 33124 \\ Ist für einen Fabrikbetrieb der Anschluß an ein Elektrizitätswerk oder eine eigene Kraftanlage vorzuziehen?}

\author{
Von Dr. Walter Straus, Charlottenburg.
}

\begin{abstract}
Übersicht In den letzten Jahren ist es den Elektrizitätswerken immer mehr gelungen, größere Abnehmer, darunter besonders Fabriken, zum Anschluß zu bewegen. Um zu zeigen, in welchen Fällen es sich für eine Fabrik empfiehlt, von einer Zentrale Strom zu beziehen, wurden Betriebskostenberechnungen für drei Beispiele durchgeführt.

Das erste Beispiel behandelt eine kleine Fabrikmit einem maximalen Kraftbedarf von ca $15 \mathrm{~kW}$, das zweite eine mittlere Fabrik mit ca $110 \mathrm{~kW}$ und das dritte eine größere mit ca $400 \mathrm{~kW}$ maximalem Kraftbedarf. Die Grundlagen der Rechnungen werden angegeben und die jährlichen Betriebskosten bei Eigenbetrieb für verschiedene Kraftmaschinenarten und bei Strombezug für verschiedene Elektrizitätswerke in Abhängigkeit von variablen Belastungen und Betriebszeiten ermittelt. Die Resultate der in Tabellenform gebrachten Rechnungen werden graphisch dargestellt, und es zeigt sich, daß der Strombezug in sehr vielen Fällen der Eigenerzeugung vorzuziehen ist.
\end{abstract}

Bis vor einigen Jahren kam für eine Fabrik zur Erzeugung der Betriebskraft selten etwas anderes als eine eigene Anlage in Frage, seitdem aber haben sich sehr viele Werke an Elektrizitätszentralen zur Lieferung der Energie angeschlossen. Die Ursache für diese Bewegung dürfte in folgenden Punkten zu suchen sein: Einmal haben sich in den letzten Jahren die Erzeugungskosten der Elektrizitätswerke bedeutend verringert, da die benutzten Maschineneinheiten an Größe und Wirkungsgrad zugenommen haben. In den gewaltigen Dampfturbinen der jüngsten Zeit läßt sich natürlich die Energie viel billiger produzieren als in den alten "Kohle fressenden" Dampfmaschinen. Auch die Anlagekosten von solchen Zentralen sind meist viel niedriger, auf die Einheit der Leistung bezogen, als diejenigen von Einzelanlagen in Fabriken. Außerdem fallen die mög- licherweise auftretenden Maximalansprüche der einzelnen Fabriken an Energie (die sogenannten Bedarfsspitzen), für welche in den Einzelanlagen Maschinensätze bereitgestellt sein müssen, zeitlich durchaus nicht zusammen, und die in der Zentrale vorhandenen Maschinen müssen nur einen Bruchteil dieses Maximalbedarfs leisten. Auch dadurch ermäßigen sich die aufzuwendenden Anlagekosten um einen bedeutenden Teil. Schließlich ist die durchschnittliche Ausnutzung einer größeren Elektrizitätszentrale weit besser als die von Einzelanlagen, so daß auch die durchschnittlichen Kosten für die benötigten Betriebsmittel sowie für Bedienung und Verwaltung nicht unwesentlich niedriger werden. Anderseits verbessert der Anschluß von Fabriken und ähnlichen willkommenen Großabnehmern den Ausnutzungsfaktor der Elektrizitätswerke noch mehr, so daß auch dadurch die relativen Selbstkosten reduziert werden. Diesen kostenmindernden Elementen sind als Verteuerung die evtl. Kosten der Leitungen und der Umformung des elektrischen Stromes gegenüberzustellen.

In einer großen Zahl von Fällen wird es daher den Elektrizitätswerken möglich sein, Fabriken die Betriebskraft billiger zu liefern, als diese sie sich selbst herstellen könnten. DaB dies nicht immer der Fall ist, liegt teils an kurzsichtigen Tarifen der betr.Zentralen und manchmal auch an den Betriebsbedingungen der Fabriken, die für den eigenen Kraftbetrieb so günstig sind, daß das Elektrizitätswerk nicht damit konkurrieren kann. Wenn daher eine Fabrik vor der Frage steht, ob sie sich an ein Elektrizitätswerk anschließen oder die Energie selbst erzeugen soll (sei es, daß die alte Kraftzentrale zu unwirtschaftlich arbeitet oder nicht mehr 
ausreicht, oder daß es sich um eine Neugründung handelt), so ist es notwendig, eine eingehende Rentabilitätsberechnung für die verschiedenen Möglichkeiten anzustellen. Im folgenden sollen nun für einige Fälle solche Rechnungen ausgeführt werden; zwar wird es nicht möglich sein, ein allgemein gültiges Resultat zu erzielen, doch läßt sich aus diesen Einzelfällen ersehen, in welcher Richtung derartige Betrachtungen anzustellen und welche Ergebnisse zu erwarten sind.

Für den Antrieb der Arbeitsmaschinen einer Fabrik kommen folgende Möglichkeiten in Betracht:

1. Eigene Kraftmaschine, Antrieb durch Transmission.

2. Eigene Kraftmaschine mit elektrischem Generator, Gruppenantrieb durch größere Elektromotoren.

3. Eigene Kraftmaschine mit elektrischem Generator, Einzelantrieb der größeren Arbeitsmaschinen, Gruppenantrieb der kleineren.

4. Anschluß an Elektrizitätswerk, großer Elektromotor, Antrieb durch Transmission.

5. Anschluß an Elektrizitätswerk, Gruppenantrieb der Arbeitsmaschinen durch größere Elektromotoren.

6. Anschluß an Elektrizitätswerk, Einzelantrieb der größeren Arbeitsmaschinen, Gruppenantrieb der kleineren.

Für kleinere und mittlere Fabriken ist die erste Art des Antriebs bei eigener Krafterzeugung am gebräuchlichsten, für größere Fabriken die zweite und dritte, während bei Anschluß an ein Elektrizitätswerk im allgemeinen nur Betriebe mit kleinem Bedarf die Antriebsart 4 wählen, die das Gegenstück zu 1 darstellt, und alle größeren Werke Gruppen- bzw. Einzelantrieb bevorzugen. Hier treten nun zuerst die oft ventilierten und in unzähligen Artikeln besprochenen Fragen auf, ob Einzelantrieb oder Gruppenantrieb, ob elektrische oder mechanische Transmission vorzuziehen sei. In diesem Zusammenhange soll darauf nicht eingegangen werden, da die Ansicht der meisten Betriebsleute darüber heute wohl geklärt sein dürfte: nämlich mechanische Transmission höchstens bis zu $70 \mathrm{bzw} .120 \mathrm{~kW}$, Einzelantrieb nur in selteneren Fällen, z. B. für größere und schwerere Arbeitsmaschinen, und im allgemeinen Gruppenantrieb zu verwenden. Die Energiemenge, die eine mechanische Transmission verschlingt, ist ziemlich erheblich, besonders, wenn diese, wie in vielen Fabriken, längere Zeiten nur wenig belastet laufen muß, dagegen machen auch bei Gruppenantrieb die Verluste in den elektrischen Antriebsmotoren und deren Zuleitungen ziemlich viel aus, so daß in den folgenden Rechnungen der Einfachheit halber der Kraftbedarf beider Antriebsarten gleichgesetzt werden soll. In Wirklichkeit muß bei eingehenden Untersuchungen auch dieser Verlust berücksichtigt werden, und es zeigt sich oftmals, daß die elektrische Transmission meistens der mechanischen überlegen ist. Man findet daher auch nur recht selten in bedeutenderen Fabriken aus den letzten Jahren größere mechanische Transmissionsanlagen.

Für die Vergleichsrechnungen im folgenden sind drei Fälle gewählt worden, welche in ihrer Eigentümlichkeit einen großen Teil der in der Praxis vorkommenden Möglichkeiten charakterisieren. Als erstes Beispiel soll eine kleinere Fabrik behandelt werden, die einen Maximalbedarf von ca $15 \mathrm{~kW}$ hat, der bei Eigenbetrieb durch eine Kraftmaschine und bei Anschluß an eine Zentrale durch einen Elektromotor gedeckt wird. Eine mechanische Transmission überträgt die Leistung auf die Arbeitsmaschinen. Ähnlich wie hier werden die Verhältnisse bei vielen kleineren Werkstätten liegen. Das zweite Beispiel stellt eine größere Fabrik vor, die maximal etwas mehr als $100 \mathrm{~kW}$ benötigt. Hier soll eine Kraftmaschine (Eigenbetrieb) von ungefähr $110 \mathrm{~kW}$ Leistung, die auf eine mechanische Transmission arbeitet, mit elektrischem Gruppenantrieb (Anschluß an ein Elektrizitätswerk) von ungefähr dem genannten Maximalbedarf verglichen werden. Da die maximal benötigten Leistungen der einzelnen Gruppenmotoren niemals ganz zusammenfallen, so ist dieses Bedarfsmaximum geringer als die Gesamtleistung der angeschlossenen Motoren und entspricht ungefähr der Leistung der Kraftmaschine mit der erwähnten Annahme, daß die Transmissionsverluste annähernd ebenso hoch wie die Verluste in den Elektromotoren und Zuleitungen sind. Der dritte Fall bringt einen Vergleich für eine große Fabrik mit ca $400 \mathrm{~kW}$ Maximalkraftbedarf, die entweder eine eigene Zentrale mit drei Kraftmaschinensätzen und Generatoren zu je $200 \mathrm{~kW}$ Leistung (davon einer zur Reserve) besitzt, oder den insgesamt benötigten Strom von einem Elektrizitätswerk bezieht. Beispiel 2 und 3 dürften für den Typ einer mittleren und einer größeren Fabrik als Durchschnitt ungetähr richtige Resultate liefern.

Als Antriebsmaschine für diese drei Fälle müssen aus den vielen uns heute zur Verfügung stehenden Arten von Kraftmaschinen die passenden ausgewählt werden. Welche Type in jedem Falle die richtigste sein wird, hängt von 
einer großen Anzahl von Umständen ab, von denen mehrere bei allgemeinen Berechnungen nicht berücksichtigt werden können, z. B. von örtlichen Verhältnissen, dem Vorhandensein von billigen Betriebsmitteln, von der Notwendigkeit, größere Mengen Dampf bzw. warmes Wasser zur Verfügung zu haben. Es ist daher nur möglich, in den folgenden Berechnungen von allgemeinen Voraussetzungen auszugehen. Um einen wirtschaftlichen Überblick über die einzelnen möglichen Kraftmaschinen und einen Anhalt für die Wahl des geeigneten Antriebs zu geben, wurden für Fall 1 zwei Schaubilder entworfen, welche alle in Frage kommenden Maschinenarten, Dampfmaschine, Dampflokomobile, Leuchtgas-, Benzin- und Benzolmotor, Sauggasmaschine und Dieselmaschine berücksichtigen. Es zeigt sich hieraus, da $\beta$ bei einer Eigenanlage im allgemeinen für solch kleinen Betrieb nur Verbrennungskraftmaschinen ökonomisch sind (falls nicht Dampf in der Fabrikation benötigt wird oder sehr billige Abfallbrennstoffe zur Verfügung stehen). Infolge der mangelnden absoluten Betriebssicherheit sowie ihrer sonstigen schlechten Eigenschaften werden auch die Sauggasmotoren in den letzten Jahren viel seltener benutzt, so daß für kleinere Anlagen hauptsächlich Benzin-, Benzol-, Leuchtgas- und Dieselmotoren in Frage kommen, während für die größeren Fabriken meist Lokomobilen oder Dieselmaschinen als wirtschaftlichste Antriebsmotoren gewählt werden. Die genannten Maschinentypen werden daher auch bei den Vergleichen verwendet.

Als Vergleichsgrundlage bei den Rentabilitätsberechnungen sollen die jährlichen Betriebskosten in Mark in Abhängigkeit von der Betriebszeit dienen, die für die betreffende Fabrik bei einer eigenen Kraftanlage bzw. bei Strombezug aus einer Zentrale entstehen. Diese setzen sich zusammen aus den von den Anlagekosten herrührenden Werten für Verzinsung, Abschreibung, Versicherung und Steuern sowie aus den Kosten für Betriebsmittel, für Schmierund Putzmaterial, für Kühl- und Speisewasser, für Reparaturen und Instandhaltung sowie für Bedienung.

Die Anlagekosten der Maschinen wurden nach den Angaben der in Frage kommenden Firmen unter Berücksichtigung der üblichen Rabatte bestimmt. Sie enthalten allen Zubehör, Fundamente, Montagekosten, Rohrleitung usw. Aus dem benötigten Raume wurden die Gebäudekosten berechnet unter der Annahme, daß der Quadratmeter bebaute Fläche sich inkl. Gebäudekosten auf ungefähr $100 \mathrm{M}$ im Durchschnitt stellt. Da die Grundstückspreise überall verschieden sind, wird dieser Wert natürlich nur selten genau zutreffen, auf kleinen Plätzen wird er niedriger, in Großstädten höher sein, doch ist er als Mittel ganz gut anzunehmen. Die Abschreibungen hängen von der Nutzungsdauer der Anlage $a b$ und sind natürlich für Gebäude und Maschinen verschieden. Da die Grundstücke nicht abgeschrieben werden müssen und auch die Gebäude eine recht lange Lebensdauer haben, so genügt dafür ein Abschreibungssatz von $2 \%$. Bei Maschinen, u. zw. sowohl bei elektrischen wie Kraftmaschinen, darf man mit einerLebensdauer von ungefähr 12 bis 15 Jahren rechnen. Nach dieser Zeit sind sie allerdings noch sehr oft zu gebrauchen, doch ist es wahrscheinlich, daß sie infolge von Betriebsänderungen und Vergrößerungen sowie den Fortschritten der Technik, die bis dahin viel wirtschaftlichere Maschinen auf den Markt bringen wird, dann nur noch Altwert haben. Der gewählte Abschreibungssatz von $6,5 \%$, der nicht vom jeweiligen Buch-, sondern vom Anschaffungswert genommen werden muß, dürfte daher mittleren Verhältnissen entsprechen. Bei fast allen Maschinentypen, seien es nun Dampf-, Gas-, Ol- oder Elektromaschinen, läßt sich dieser Satz anwenden, denn ihre Lebensdauer wird ungefähr die gleiche sein. Nur für die schnellaufenden Verbrennungsmotoren wurden $7,5 \%$ für richtig gehalten, da sie sich verhältnismäßig schnell abnutzen.

Für Verzinsung des Anlagekapitals wird im allgemeinen bei derartigen Vergleichsrechnungen ein viel zu niedriger Prozentsatz eingesetzt. Da einer der Hauptvorteile des Anschlusses einer Fabrik an ein Elektrizitätswerk der ist, daß der Unternehmer die teuren Anlagekosten der eigenen Erzeugungsanlage spart, so muß immer mit dem Prozentsatz gerechnet werden, mit dem sich das Anlagekapital einer Fabrik im allgemeinen verzinst. Dieser Satz verringert sich nur dadurch, daß ein Unternehmer einen Teil seines notwendigen Betriebskapitals zu einem billigeren Satze, ungefähr zu 4,5 bis $5 \%$ als Hypothek oder Obligationsanleihe bekommen kann. Da er jedoch sein eigenes Kapital zu mindestens 6,5 bis $7,5 \%$ verzinst haben will (andernfalls wird er sich nur schwer zu einer Mehrausgabe entschließen), und da das billiger erhaltene Kapital selten mehr als $30 \%$ des Gesamtkapitals ausmacht, so dürfte ein Durchschnittszinssatz von $5,5 \%$, wie er hier gewählt wurde, gewiß nicht zu hoch sein. Die Kosten für Instandhaltung und Reparaturen der Anlage werden im allgemeinen in Prozenten des Anlagekapitals ausgedrückt und hängen von der Ausnutzung der Maschinen ab. In Utbereinstimmung mit den in der Praxis üblichen Annahmen wurde derProzentsatz beiVerbrennungskraftmaschinen und Lokomobilen je nach der Benutzungsdauer zwischen 1 und $3 \%$, letzterer 
Wert bei 6000 Std, jährlicher Betriebszeit, für elektrische Maschinen bis $0,5 \%$ und für Gebäude zu $0,5 \%$ in den Rechnungen angenommen.

Die Betriebsmittelkosten der Maschinen hängen $a b$ von der Belastung und dem technischen Wirkungsgrade derselben sowie von den Kosten der Energieeinheit in dem betreffenden Energieträger und der Betriebsdauer. Um sie ganz genau für eine bestimmte Anlage zu berechnen, wäre es nötig, eine Belastungskurve derselben aufzustellen und danach eine Verbrauchskurve zu konstruieren, aus der sich dann durch Planimetrierung der Gesamtverbrauch ergibt. Dies würde jedoch für die hierangestellten Rechnungen zu kompliziert sein und müßte sich auch nur auf einige ganz bestimmte Fälle beschränken. Deshalb wurden drei verschiedene Belastungsfälle angenommen, $1 / 4,1 / 2$ und $3 / 4$ durchschnittliche Belastung und fürverschiedene jährliche Benutzungszeiten derAnlage die Kosten ermittelt. Diese werden dann in Abhängigkeit von der Betriebszeit bzw. den an die Arbeitsmaschinen abgegebenen Kilowattstunden Energie in Schaubildern, getrennt für die verschiedenen Maschinengrößen, aufgetragen. Die auf diese Weise erzielten Kurven dürften ein ziemlich zuverlässiges Vergleichsmaterial für alle möglichen Fälle liefern. Dabei ist der Fall, daß die Maschinen immer voll belastet laufen, mit Absicht weggelassen, denn in der Praxis kommt so etwas niemals vor. Wenngleich die für den Betrieb einer Fabrik benötigten Kraftmaschinen im allgemeinen nicht größer gewählt werden, als dem maximal auftretenden Kraftverbrauch entspricht, abgesehen von Reserven, so ist in den meisten Fabriken die Kraftanlage im Jahre durchschnittlich mit nicht mehr als $50 \%$ dieses Maximums belastet. Dies erklärt sich daraus, daß zeitweise nur wenige Arbeitsmaschinen mit voller Kraft arbeiten, zeitweise aber auch zufällig gerade mehrere schwere Maschinen gleichzeitig in voller Tätigkeit sind, wie dies selbst bei gleichbleibender Konjunktur die Regel bildet. (Daß einmal sämtliche Arbeitsmaschinen im gleichen Moment den vollen Energiebedarf haben, ist unmöglich. Daher stellt das eben erwähnte Maximum des Energiebedarfs nur einen Teil der Energiesumme sämtlicher Arbeitsmaschinen dar.) In verschiedenen Veröffentlichungen und Statistiken (z. B. ,ZZeitschrift des Vereins deutscher Ingenieure " 1913, S. 1040) wurde festgestellt, daß in den meisten Fabriken die jährliche Durchschnittsbelastung des Maximums zwischen 30 und $50 \%$ beträgt. Diese Zahlen mögen einigen Betriebsleuten vielleicht unglaublich niedrig vorkommen, doch liegen sie einwandfrei fest und sind jederzeit nachzuprüfen. Sie spielen natürlich bei der hier zu behandelnden Frage eine große Rolle. Es wäre sehr zu wünschen, daß möglichst viele Fabrikleiter solche Zahlen sammeln und veröffentlichen wollten. (In elektrischen Anlagen lassen sie sich mittels eines registrierenden Belastungsmessers sehr leicht feststellen.)

Die bei den später folgenden Rechnungen benutzten Betriebsmittelverbrauchszahlen beziehen sich auf die vom Motor bzw. Generator abgegebeneKilowattstundenleistung (abgekürzt kW Std).

Für Benzinmotor $\left.{ }^{1}\right), 15 \mathrm{~kW}$, Schnelläufertype, stehend, wurden folgende Werte angenommen: als Preis von $100 \mathrm{~kg}$ Benzin $35 \mathrm{M}$ (dürfte momentan wohl etwas zu niedrig sein), Verbrauch bei $1 / 4$ Belastung pro kW Std $0,90 \mathrm{~kg}$, bei $1 / 2$ Belastung $0,64 \mathrm{~kg}$, bei $3 / 4$ Belastung $0,54 \mathrm{~kg}$.

Benzolmotor, $15 \mathrm{~kW}$, Schnelläufertype, stehend: Preis von $100 \mathrm{~kg}$ Benzol $30 \mathrm{M}$, Verbrauch bei $1 / 4$ Belastung pro $\mathrm{kW}$ Std $0,87 \mathrm{~kg}$, bei $1 / 2$ Belastung $0,59 \mathrm{~kg}$, bei $3 / 4$ Belastung $0,50 \mathrm{~kg}$.

Leuchtgasmotor, Schnelläufertype, stehend, $15 \mathrm{~kW}$ : Preis von $1 \mathrm{~m}^{3}$ Leuchtgas $10 \mathrm{Pf}$ Verbrauch pro $\mathrm{kW}$ Std ab Uhr bei $1 / 4$ Belastung $2,08 \mathrm{~m}^{3}$, bei $1 / 2$ Belastung $1,43 \mathrm{~m}^{3}$, bei $3 / 4 \mathrm{Be}-$ lastung $1,20 \mathrm{~m}^{3}$.

Leuchtgasmotor, liegend, Langsamläufer, $15 \mathrm{~kW}$ : Verbrauch pro $\mathrm{kW}$ Std ab Uhr bei $1 / 4$ Belastung $1,48 \mathrm{~m}^{3}$, bei $1 / 2$ Belastung $1,0 \mathrm{~m}^{3}$, bei $3 / 4$ Belastung $0,86 \mathrm{~m}^{3}$.

Dieselmotor, $15 \mathrm{~kW}$, betrieben mit Gasöl, liegend: Preis des Gasöls pro $100 \mathrm{~kg} 11 \mathrm{M}$, Verbrauch pro $\mathrm{kW}$ Std bei $1 / 4$ Belastung $0,44 \mathrm{~kg}$, bei $1 / 2$ Belastung $0,345 \mathrm{~kg}$, bei $3 / 4$ Belastung $0,315 \mathrm{~kg}$.

Dieselmotor, $110 \mathrm{~kW}$, stehend, betrieben mit Teeröl: Preis des Teeröls pro $100 \mathrm{~kg} 5 \mathrm{M}$, Verbrauch an Teeröl pro kW Std bei $1 / 4 \mathrm{Be}$ lastung $0,430 \mathrm{~kg}$, bei $1 / 2$ Belastung $0,340 \mathrm{~kg}$, bei $3 / 4$ Belastung $0,300 \mathrm{~kg}$, dazu ein bei allen Belastungen gleichbleibender Zuschlag von Gasöl zum Zünden von $1,5 \mathrm{~kg} /$ Motor Std.

Dieselmotor mit Generator, $200 \mathrm{~kW}$ elektrischer Leistung, Teerölbetrieb: Verbrauch pro abgegebene $\mathrm{kW}$ Std des Generators bei $1 / 4 \mathrm{Be}$ lastung (Generatorwirkungsgrad $85 \%$ ) $0,505 \mathrm{~kg}$, bei $1 / 2$ Belastung (Generatorwirkungsgrad $89,5 \%$ ) $0,380 \mathrm{~kg}$, bei $3 / 4$. Belastung (Generatorwirkungsgrad $91,5 \%$ ) $0,328 \mathrm{~kg}$, dazu ein Zündölzusatz von $3 \mathrm{~kg} /$ Motor Std.

2) Die im folgenden angegebenen Verbrauchswerte dïrften vielen etwas hoch erseheinen. doch sind sie an Hand des kürzlich erschienenen Buches von [B a r t h: „Wahl, Proiektierung nndBetrieh vonKraftanlagen. derbekanntlich Autorität auf diesem Gebiete ist, und nuch Ergehnissen der Praxis ermittelt. Diese Zahlen sind meist etwas höher als fabriken angeben, da letztere im praktischen Betrieb nie erreicht werden. 
Heißdampflokomobile mit Kondensation, $110 \mathrm{~kW}$ Dauerleistung: Preis von $100 \mathrm{~kg}$ Steinkohle von 7500 Wärmeeinheiten 2,20 M (Durchschnittswert von Berlin, Hannover und Rheinland), Verbrauch pro $\mathrm{kW}$ Std bei $1 / 4 \mathrm{Be}$ lastung $1,3 \mathrm{~kg}$, bei $1 / 2$ Belastung $1,06 \mathrm{~kg}$, bei $3 / 4$ Belastung $0,96 \mathrm{~kg}$, dazu ein bei allen $\mathrm{Be}$ -
kleinenMaschinen ziemlich unbedeutend. Nimmt man an, daß die Maschinenwärter in den Zeiten, in denen sie für ihre Maschinen nichts zu tun haben, anderweitig beschäftigt werden, und da $B$ der Stundenlohn eines solchen Mannes ca 60 bis $70 \mathrm{Pf}$ beträgt, so ergeben sich folgende Kosten in Mark pro Jahr:

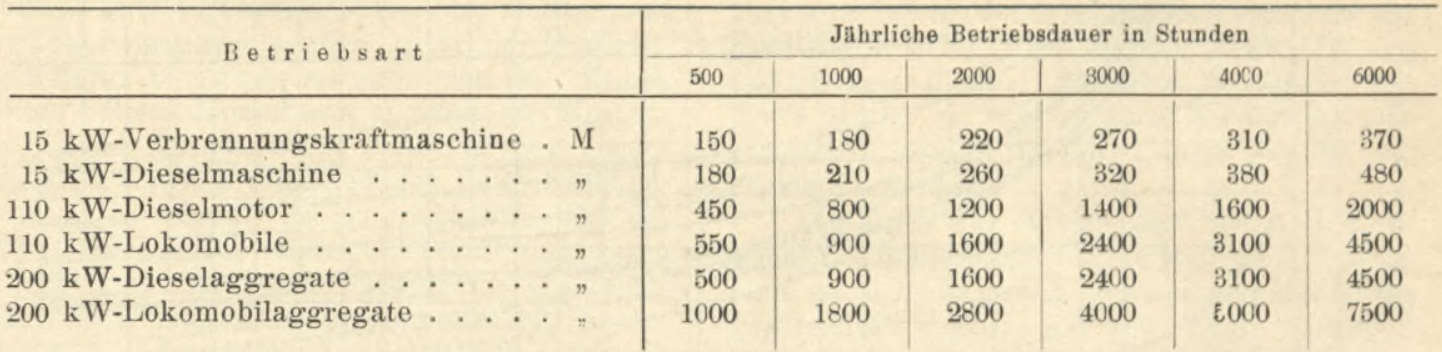

lastungen gleichbleibender Zuschlag von 105 $\mathrm{kg} / \mathrm{Tag}$ für Anheiz- und Stillstandsverluste.

Heißdampflokomobile (mit Kondensation) mit Generator, $200 \mathrm{~kW}$ elektrische Leistung: Verbrauch pro abgegebene $\mathrm{kW}$ Std des Generators, bei Annahme derselben Generatorwirkungsgrade wie bei dem gleichgroßen Dieselaggregat (siehe oben) bei $1 / 4$ Belastung $1,38 \mathrm{~kg}$, bei $1 / 2$ Belastung $1,07 \mathrm{~kg}$, bei $3 / 4$ Belastung $0,97 \mathrm{~kg}$, dazu ein bei allen Belastungen gleichbleibender Zuschlag von $186 \mathrm{~kg} / \mathrm{Tag}$ für Anheizund Stillstandsverluste.

Die hier angegebenen Verbrauchszahlen entstammen den Angaben der Praxis und unterscheiden sich oft nicht unwesentlich von den sogenannten ,Garantiewerten“, die nur auf dem Versuchsstande oder bei besonders ausgebildeter Bedienung, dagegen nie im praktischen Betriebe erreicht werden können.

Die Ausgaben für Schmieröl hängen nicht von der Belastung, sondern von den Betriebsstunden der Maschinen ab und wurden nach praktischen Erfahrungen, wie folgt, in die Rechnungen eingesetzt: Preis des Schmieröles 50 bis $60 \mathrm{M} / 100 \mathrm{~kg}$, Verbrauch der $15 \mathrm{~kW}$-Verbrennungskraftmaschinen $160 \mathrm{~g}$, der $15 \mathrm{~kW}$-Dieselmaschine $200 \mathrm{~g} /$ Betriebs Std, Verbrauch der $110 \mathrm{~kW}$-Dieselmaschine und -Lokomobile $600 \mathrm{~g}$ pro Betriebs Std, Verbrauch der $200 \mathrm{~kW}$-Aggregate $1000 \mathrm{~g} /$ /Betriebs Std. Für Putz- und Packungsmaterial wurden bei den $15 \mathrm{~kW}-\mathrm{Ma}$ schinen $0,085 \mathrm{Pf}$, bei den von $110 \mathrm{~kW} 0,055 \mathrm{Pf}$, bei den $200 \mathrm{~kW}$-Einheiten $0,04 \mathrm{Pf} / \mathrm{kW}$ Std gerechnet.

Die Kosten für Bedienung sind je nach dem betreffenden Ort verschieden. Die bei den Rechnungen verwandten Werte entsprechen daher mittleren Angaben. Sie hängen von der jährlichen Betriebsstundenzahl ab und sind bei den
Die letzten beiden Reihen gelten für den Fall, daß zwei Aggregate in Betrieb sind, wie es für Beispiel 3 anzunehmen ist.

Die Kosten des Kühlwassers können nur sehr oberflächlich angegeben werden. Da sie aber anderseits nicht vernachlässigt werden dürfen, so wurden als Mittelwerte ${ }^{1}$ ) in den Tabellen folgende Zahlen eingesetzt: Preis von $1 \mathrm{~m}^{3}$ Wasser $10 \mathrm{Pf}$, Verbrauch der $15 \mathrm{~kW}$-Diesel-, Leuchtgas-, Benzin- und Benzolmaschinen $30 \mathrm{l}$ pro kW Std, der größeren Dieselmaschinen 18 und $15 \mathrm{l} / \mathrm{kW}$ Std. Für die Lokomobilen, welche ca 250 bis $400 \mathrm{l} / \mathrm{kW} \mathrm{Std}$ gebrauchen, wurde angenommen, daß Flußwasser zur Verfügung steht oder daß eine kleine Rückkühlanlage vorhanden ist. Das Kühl- und Speisewasser kostet dann ungefähr für die $110 \mathrm{~kW}$-Lokomobile $200 \mathrm{M}$ pro Jahr bei jeder Belastung und für die 200 $\mathrm{kW}$-Lokomobile ca $350 \mathrm{M}$.

Die übrigen Betriebsausgaben, Versicherungs- und Revisionskosten, Steuern und Abgaben sind für alle Betriebe und Landesteile so verschieden, daß es unmöglich ist, einen einigermaßen im Mittel zu treffenden Wert dafür einzusetzen. Dieser Teil der Betriebskosten wurde daher weggelassen, da außerdem die dafür aufzuwendenden Beträge recht gering sind.

Die vorstehend angegebenen Zahlen, auf welchen die Vergleichsrechnungen basieren, sind natürlich nur Mittelwerte und machen nicht den Anspruch auf absolute Gültigkeit. Da sie jedoch für viele Fälle annähernd zutreffen werden, so können sie ein ungefähres Bild der wirklichen Verhältnisse geben.

In den Abb. 1 und 2 ist nun ein Vergleich der $15 \mathrm{~kW}$-Kraftmaschinen mit einem gleich-

1) In Übereinstimmung mit Friedrich Barth, ,Wahl, Projektierung und Betrieb von Kraftanlagen", und U'rbahn: Reutlinger,..Ermittelung der billigsten Betriebskraft für Fabriken". 


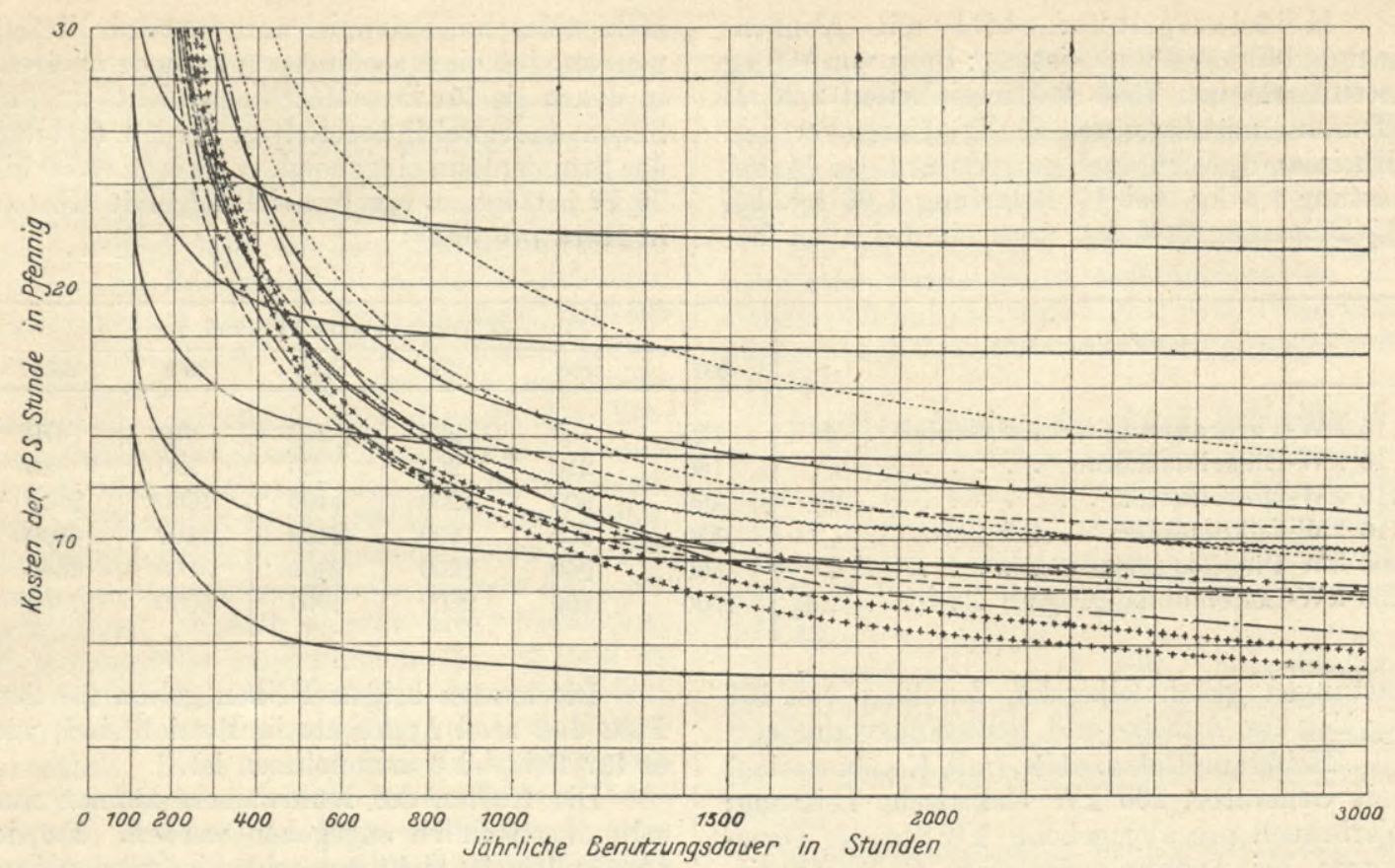

Abb. 1. Kostenvergleich der $15 \mathrm{~kW}$-Kleinkraftmaschinen. Vollbelastung.

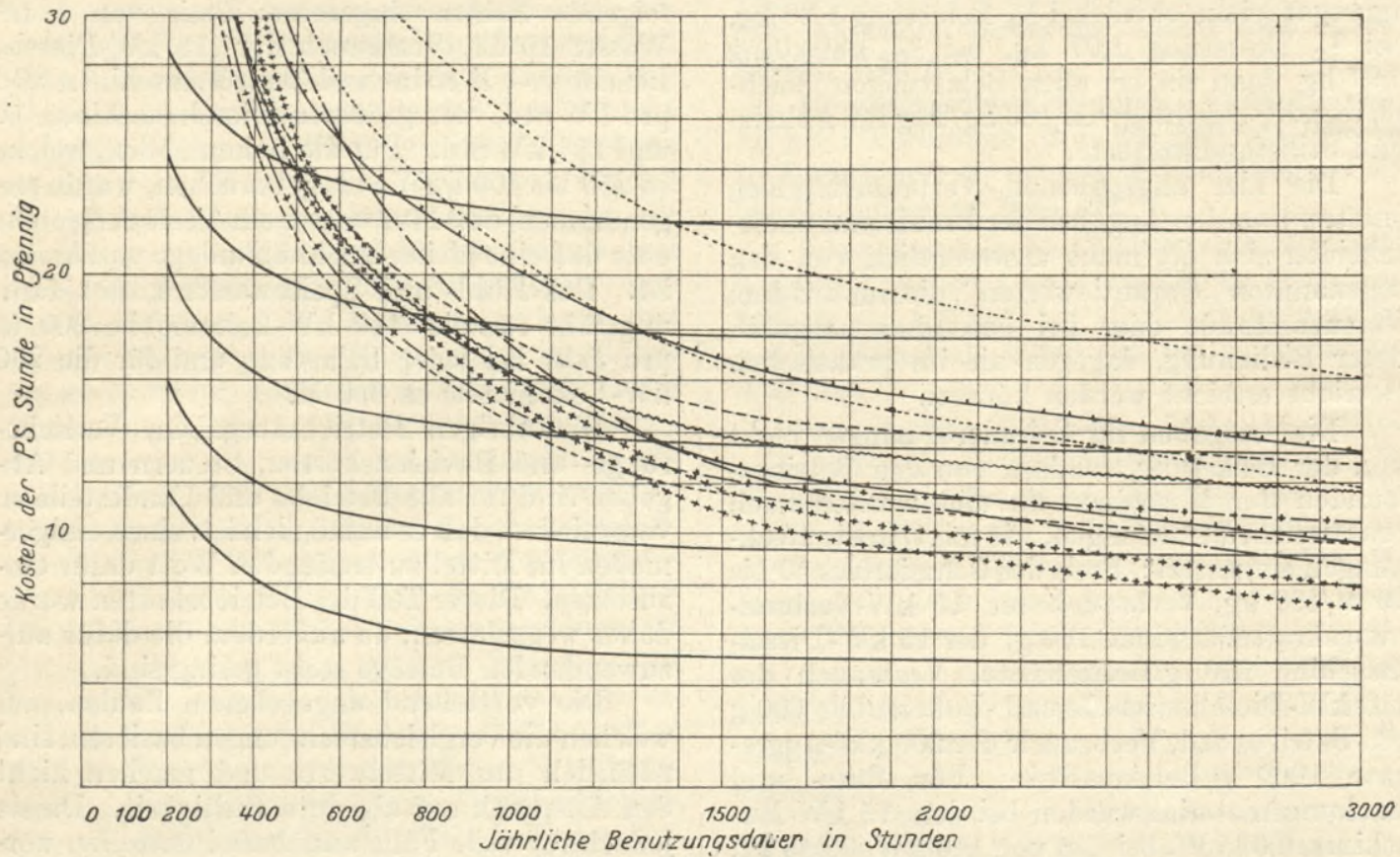

Abb. 2. Kostenvergleich der $15 \mathrm{~kW}$-Kleinkraftmaschinen. Ein Drittel Betriebszeit Vollbelastung; zwei Drittel Betriebnzeit Halbbelastung.

Zeichenerklärung für Abb. 1 und 2.

- Elektromotor (1 kW Std 25, 20, 15, 10, 5 Pf).

- - D Dieselmotor (100 kg Gasöl $11 \mathrm{M})$.

-.. Leuchtgasmotor ( $1 \mathrm{~m}^{2}$ Gas 15 und $10 \mathrm{Pf}$ ).

Dampfmaschine (100 kg Steinkohlen 3 und $1.5 \mathrm{M}$ ).

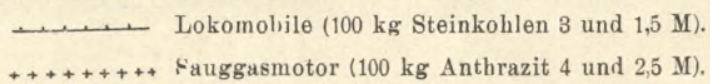

Benzinmotor (100 kg Benzin $24 \mathrm{M}$ ).

- + + - Benzolmotor (100 kg Benzol $21 \mathrm{M}$ ). 
starken Elektromotor durchgeführt, bezogen auf den aus den Gesamtjahreskosten ermittelten Durchschnittspreis der geleisteten Pferdekraftstunde, in Abhängigkeit von den jährlichen Betriebsstunden. Die hierbei gemachten Voraussetzungen ${ }^{\mathbf{1}}$ ) sind etwas anders als sie vorstehend angegeben wurden. So gilt das erste Kurvenblatt für Vollbelastung der Motoren, während für das zweite in einem Drittel der Betriebszeit Vollbelastung und in zwei Drittel der Betriebszeit halbe Belastung angenommen ist. Zweck dieser Schaubilder ist nur, ungefähr das Kostenverhältnis der einzelnen Motorentypen gegeneinander und bei verschiedenen Betriebsmittelpreisen zu zeigen. Es ist ersichtlich, $\mathrm{da} \beta$ der Elektromotor bei niedrigen Benutzungszeiten selbst noch für hohe Strompreise gegenüber allen Kraftmaschinen konkurrenzfähig bleibt, daß aber anderseits bei den höherenBenutzungszeiten die Strompreise schon recht niedrig sein müssen, damit nicht eigener Kraftmaschinenbetrieb vorzuziehen wäre. Die Belastungsverhältnisse im Schaubilde 1 sind allerdings nur ideell und entsprechen nicht der Wirklichkeit. Für schlechtere Durchschnittsbelastung, wie sie die Regel bildet, schneidet der Elektromotor viel besser ab.

Im Gegensatz zu diesen angenäherten Resultaten wurden genauere Betriebskostenberechnungen in Zahlentafeln zusammengestellt, welche sich auf die oben erwähnten Annahmen stützen. In den Tabellen 1 bis 5 sind die jährlichen Betriebskosten für je einen LeuchtgasSchnelläufer, Leuchtgas-Langsamläufer, Benzin-, Benzol- und Dieselmotor von $15 \mathrm{~kW}$ Leistung bei durchschnittlich $1 / 4,1 / 2$ und $3 / 4 \mathrm{Be}$ lastung und bei verschiedenen Betriebszeiten angegeben (Beispiel 1).

In den Tabellen 6 und 7 werden die Betriebskostenrechnungen für einen $110 \mathrm{~kW}$ Dieselmotor und eine $110 \mathrm{~kW}$-Heißdampflokomobile unter den gleichen Voraussetzungen durchgeführt (Beispiel 2).

In den Tabellen 8 und 9 werden die Kosten für eine Dieselmotoren- und eine Heißdampflokomobilen-Fabrikzentrale (Beispiel 3) angegeben; es sind drei Aggregate zu je $200 \mathrm{~kW}$ aufgestellt, von denen eines als Reserve dient. Natürlich werden hier die Kosten verhältnismäßig ebenso groß wie bei Abb. 2, da die Abschreibung und Verzinsung des Reserveaggregates und der elektrischen Generatoren sowie der Leistungsverbrauch der letzteren noch zu den Kraftmaschinenkosten kommen.

1) Die genauen Berechnungen, welche diesen beiden Schaubildern zu Grunde gelegt wurden, sind in dem im Verlage von Franz Siemenroth. Berlin, erschienenen Buche des Verfassers, Die deutsohen Ưberland-Zentralen" zu finden (vgl. auch „ETZ” 1913, S. 472).
Den im ersten Teil dieser Arbeit durchgeführten Rechnungen für die eigenen Kraftanlagen folgen nun die Aufstellungen der Betriebskosten für den Anschlu B an eine Elektrizitätszentrale. Diese setzen sich zusammen aus den Abschreibungs-, Verzinsungs-, Instandhaltungs- und Bedienungskosten der an Stelle eigener Kraftmaschinen verwandten Elektromotoren, den Schmierölkosten sowie der Elektrizitätszählermiete und den Kosten für den elektrischen Strom. Letzterer Punkt ist um vieles größer als alle anderen Kostenelemente zusammen, und es soll daher zuerst darauf eingegangen werden.

Die Tarife für elektrischen Strom sind für solche Großabnehmer, wie sie hier in Frage kommen, meist recht gering. Je nach der Größe des Elektrizitätswerkes und der Lage desselben unterscheiden sie sich aber auch nach Höhe und Art nicht unwesentlich. Im folgenden sind die Kraftstromtarife bzw. Großabnehmertarife einiger deutschen Industrieorte kurz zusammengestellt, welche für den hier gewählten Vergleich in Frage kommen. Meistens gehen die. selben nur bis zu einer Abnahme von 0,6 MillkW Std im Jahre. Mit größeren Abnehmern werden Sonderabmachungen vereinbart, die nicht veröffentlicht sind. In einigen Tarifen konnten jedoch auch Angaben für größere Kunden gefunden werden, und diese wurden dann in den Berechnungen benutzt.

Über die Eigenart der hier gebrachten Stromtarife ist in der einschlägigen Literatur so viel zu finden, daß es sich hier wohl erübrigen dürfte, darauf einzugehen. Es wird daher genügen, die bei den Rechnungen verwandten Tarife selbst abgekürzt aufzuführen, u. zw. nur soweit, als sie hier benötigt werden:

\section{Berliner Elektricitäts-Werke.}

a) Niederspannung: In der Stadt Berlin kostet die Kilowattstunde $16 \mathrm{Pf}$, in den Vororten teilweise ebensoviel, zum größten Teil jedoch nur $10 \mathrm{bzw} .11 \mathrm{Pf}$. Der Tarif ist sehr hoch, besonders für größere Abnehmer, und wird in der nächsten Zeit ermäßigt werden.

b) Hochspannung: In der Stadt kostet die Energie bei einem jährlichen Mindestverbrauch von $200000 \mathrm{kWStd}$ und Mindestbenutzungsdauer von 2000 Std 10 bis $7,5 \mathrm{Pf} / \mathrm{kW} \mathrm{Std,} \mathrm{in}$ den Vororten bei Mindestverbrauch von 100000 $\mathrm{kW}$ Std und Mindestbenutzungsdauer von 2000 Std je nach Benutzungsdauer 9 bis $6,5 \mathrm{Pf} / \mathrm{kW}$ Std.

Auch diese beiden Tarife sind sehr hoch und können bei größeren Abnehmern nicht mit Selbsterzeugung konkurrieren. Doch sollen sie gleicbfalls in der nächsten Zeit herabgesetzt 
werden. Bei besonders günstigen Konsumenten werden Sonderabmachungen getroffen.

\section{Städtisches Elektrizitätswerk Köln.}

Doppeltarif: a) Preis in der Sperrzeit: bis zu $2000 \mathrm{kWStd}$ im Jahre $45 \mathrm{Pf}$, von 2001 bis $5000 \mathrm{~kW}$ Std 40 Pf usw. bis über $150000 \mathrm{~kW}$ Std $12,5 \mathrm{Pf} / \mathrm{kW}$ Std. Außerdem Rabatte.

b) Preis in der übrigen Zeit: bis zu 5000 kWStd im Jahre 16 Pf usw. bis über 1000000 kW Std 4 Pf/kW Std. Außerdem Rabatte je nach Benutzungsdauer.

\section{Städtisches Elektrizitätswerk Dort- mund.}

a) Niederspannung: Die Kilowattstunde kostet 20 bis $11 \mathrm{Pf}$ je nach Abnahmemenge.

b) Hochspannung: Die Kilowattstunde kostet 11 bis 6 Pf. Außerdem Rabatte je nach Benutzungsdauer.

4. E lektrizitätswerk Ludwigshafen a.Rh.

Für Großabnehmer kostet die Kilowattstunde 10 bis $8 \mathrm{Pf}$ außerhalb der Sperrzeit, während dieser $25 \mathrm{Pf}$.

\section{Elektrizitätswerk München-Glad- bach.}

Die Energie kostet bei jährlicher Abnahme von $10000 \mathrm{kWStd} 12 \mathrm{Pf}$ bis herab zu 4,5 Pf pro kWStd bei jährlicher Abnahme von $500000 \mathrm{~kW}$ Std. In der Sperrzeit beträgt der Preis 1 Pf mehr, nachts $1 / 2$ Pf weniger. Außerdem Rabatte je nach Benutzungsdauer.

6. Elektrizitätswerk Neukölln.

a) Kleinabnehmer: Die Kilowattstunde kostet $13 \mathrm{Pf}$.

b) Großabnehmer: Bei mehr als 36000 kW Std Jahresabnahme ist eine Grundgebühr von 8 bis $6 \mathrm{M}$ monatlich für je $1 \mathrm{~kW}$ des Maximalbedarfs, abgestuft nach dem verbrauchten Leistungsmaximum, außerdem ein Preis von $4 \mathrm{Pf} / \mathrm{kW}$ Std zu zahlen. Benutzungsdauerrabatte.

\section{Städtisches Elektrizitätswerk Nürn- berg.}

Bei mindestens $24000 \mathrm{~kW} \mathrm{Std} \mathrm{Jahresab-}$ nahme ist eine Grundgebühr von $6 \mathrm{M}$ monatlich für je $1 \mathrm{~kW}$ des Maximalbedarfs und ein Preis von 6.5 bis $4 \mathrm{Pf} / \mathrm{kW} \mathrm{Std} \mathrm{zu} \mathrm{zahlen.}$

\section{Städtisches Elektrizitätswerk Osnabrück.}

Hochspannungstarif: Für das im Jahre maximal abgenommene Kilowatt ist eine Grund- gebühr von $160 \mathrm{M}$ bei einer Benutzungsdauer bis zu 2000 Std jährlich und für die weiteren über 2000 Benutzungsstunden entnommenen Kilowattstunden je $3,0 \mathrm{Pf}$ zu zahlen. Außerdem Rabatte nach dem verbrauchten Leistungsmaximum.

\section{Rheinisch-Westfälisches Elektrizi-} tätswerk, Essen.

a) Niederspannung: Die Energie kostet bei einer jährlichen Abnahme bis zu $6000 \mathrm{~kW} \mathrm{Std}$ $14 \mathrm{Pf}$ bis herab zu $7,75 \mathrm{Pf} / \mathrm{kW} \mathrm{Std}$ bei einer jährlichen Abnahme von mindestens 120000 kW Std.

b) Hochspannung: Die Kilowattstunde kostet bei einer jährlichen Abnahme von mindestens $12000 \mathrm{kWStd}$ 11,5 Pf bis herab zu 6 Pf bei einer jährlichen Abnahme von mindestens $600000 \mathrm{~kW}$ Std. Außerdem Benutzungsdauerrabatte.

\section{Städtisches Elektrizitätswerk Saarbrücken.}

Bei einer jährlichen Mindestabnahme von $100000 \mathrm{KW}$ Std und mindestens 2000 Benutzungsstunden ist eine Grundgebühr von $70 \mathrm{M}$ für das jährlich maximal entnommene Kilowatt sowie ein Zuschlagspreis von $2,5 \mathrm{Pf} / \mathrm{kW} \mathrm{Std,}$ hochspannungsseitig gemessen, zu bezahlen.

Auf Grund dieser Tarife wurden die Stromkosten unter den nachfolgenden Annahmen ermittelt: für die drei Beispiele war der als Grundlage dienende Maximalleistungsbedarf entsprechend der Leistung der Kraftmaschinen (bei der Selbstkostenberechnung für Eigenanlagen) angenommen. Hieraus ergeben sich die für die Rabattberechnung maßgebenden Benutzungszeiten. Verschiedene der Tarife enthalten nicht mehr die Rabatte bei so hohen Benutzungszeiten, wie sie in den Beispielen teilweise überschritten werden, sowie bei Entnahme von über 1 Mill. kW Std. Es konnte daher bei solchen Werten nicht mit höheren Rabatten gerechnet werden. In Wirklichkeit gewähren die Elektrizitätswerke in diesen Fällen meist größere Ermäßigungen, und die jeweils zu bezahlenden Stromkosten sind oftmals noch niedriger als sie hier angegeben wurden. Außerdem dienen die Tarife, worauf ausdrücklich aufmerksam gemacht werden soll, nur als Grundlage für die Verhandlungen und werden sehr oft dem gerade vorliegenden Einzelfall angepaßt. Da die Sperrzeiten der einzelnen Werke ungefähr zusammenfallen, u. zw. auf die Abendstunden bis 8 oder 9 Uhr, so wurde allgemein, soweit dies nötig war, damit gerechnet, daß $10 \%$ der insgesamt abgenommenen Kilowattstunden in die Sperrzeit 
fallen. In einigen Tarifen richtet sich die Benutzungsdauer und damit der Rabatt nach dem in die Sperrzeit fallenden Maximum. Durch geschickte Besetzung seiner Maschinen kann ein Unternehmer gerade diesen Faktor leicht herabdrücken. Es wurde jedoch, um nicht zu günstige Resultate für den elektrischen Betrieb zu erhalten, der volle Wert wie bei Eigenbetrieb benutzt. In den Fällen, in denen Hochspannung bezogen werden kann, wurde ein entsprechender Betrag für die Umformungs- und Leerlaufsverluste, die ja bekanntlich nicht groß sind, berücksichtigt. In Grenzfällen der Staffeltarife wurde grundsätzlich für die Stromkosten nie ein höherer Wert eingesetzt als der niedrigsten Zahlung der nächsten Stufe entspricht, so z. B. beginnt beim Rheinisch-Westfälischen Elektrizitätswerk bei einer Abnahme von 84000 $\mathrm{kWStd}$ eine neue Stufe zu 8,50 Pf/kW Std, während die Energie bis dahin 8,75 Pf kostet. Hat der Verbraucher nun $82500 \mathrm{~kW}$ Std entnommen, so hätte er nach der niederen Stufe $7215 \mathrm{M}$ zu zahlen, während der Preis für 84000 kW Std nur $7140 \mathrm{M}$ beträgt. Dieses Verfahren wird in der Praxis oft angewandt und führt infolge der Eigentümlichkeit einiger Tarife manchmal zu merkwürdigen Gegensätzen. (So z. B. rentiert es sich für manche Lichtkonsumenten der Berliner Elektricitäts-Werke, die nur 25000 kW Std im Jahre abnehmen, 0,1 Mill. kW Std zu bezahlen, weil sie dadurch in den billigen Hochspannungstarif hineinkommen und immerhin noch ca 2000 M im Jahre sparen können.)

In Tabelle 10 sind nun die Betriebskosten einer an eine Zentrale angeschlossenen Fabrik analog Tabelle 1 bis 5 (mit denselben Belastungen, Betriebszeiten und geleisteten $\mathrm{kW} \mathrm{Std)} \mathrm{aufgetragen,} \mathrm{u.} \mathrm{zw.} \mathrm{wurden} \mathrm{die} \mathrm{Strom-}$ kosten auf Grund der oben erwähnten Tarife berechnet. Hierbei wurde angenommen, da $B$ der $15 \mathrm{~kW}$-Elektromotor, ebenso wie die gleichstarke Kraftmaschine bei Eigenbetrieb, eine Transmission treibt. Der Wirkungsgrad des Motors bei $1 / 4$ durchschnittlicher Belastung ist $79,5 \%$, entsprechend einem Verbrauch von 1,26 $\mathrm{kW}$ pro an der Motorwelle geleistetes Kilowatt. Für 1/2 Belastung sind die Werte $86 \%$ und 1,16 $\mathrm{kW}$, für $3 / 4$ Belastung $87,5 \%$,und $1,14 \mathrm{~kW}$.

In Tabelle 11 sind die Betriebskostenrechnungen für Fall 2 der Beispiele bei Anschluß an eine Zentrale aufgestellt. Wie oben bemerkt, sollen hierbei an Stelle einer Kraftmaschine von $110 \mathrm{~kW}$ Leistung drei Elektromotoren zu je ca 35 bis $40 \mathrm{~kW}$ verwandt werden. Da der Wirkungsgrad der großen Transmission bei Eigenbetrieb ungefähr dem der Elektromotoren bei Strombezug entspricht, so wurde angenommen, daf die Zentrale ebenso viele Kilowattstunden an die Fabrik abgeben müßte als nach Tabelle 6 und 7 die Kraftmaschine von $110 \mathrm{~kW}$ an die Haupttransmission liefert. Die Betriebszeiten und Belastungen sind natürlich auch dieselben wie in den Tabellen 6 und 7 .

Tabelle 12 enthält die Kosten des Strombezugs einer Fabrik nach Beispiel 3, entsprechend den Rechnungen in Tabelle 8 und 9. Da bei eigenen Kraftzentralen auch Zählerkosten zu rechnen sind, so wurden dieselben nicht berücksichtigt.

Die Resultate dieser Rechnungen sind in den Abb. 3 bis 12 graphisch aufgetragen. Abb. 3 zeigt einen Vergleich der Betriebskosten eines Dieselmotors von $15 \mathrm{~kW}$ Leistung mit einem gleichstarken Elektromotor, der an das Elektrizitätswerk Neukölln angeschlossen ist, bei verschiedenen Betriebszeiten und durchschnittlich $3 / 4$ Belastung, aufgeteilt nach den einzelnen Kostenelementen. Es erhellt hieraus die bekannte Tatsache, daß bei elektrischem Antrieb die Stromkosten den Hauptanteil an den gesamten Ausgaben bilden, bei den Kraftmaschinen dagegen die Kosten für Abschreibung und Verzinsung. So erklärt es sich, daß bei geringen Betriebszeiten der Anschluß an eine Zentrale selbst unter Annahme von hohen Stromkosten noch dem eigenen Kraftmaschinenbetriebe wirtschaftlich überlegen ist. Deutlich ist dies besonders in den Abb. 4 bis 12 zu sehen. Die Betriebskosten pro Jahr sind hier nach den Ergebnissen der Tabellen in Abhängigkeit von den jährlichen Betriebsstunden aufgetragen. Abb. 4,5 und 6 enthalten Beispiel 1 bei $1 / 4,1 / 2$ und $3 / 4$ durchschnittlicher Belastung, Abb. 7,8 und 9 Beispiel 2 und die übrigen Beispiel 3.

In fast allen hier aufgeführten Fällen wird, wie aus den Kurven ohne weiteres zu entnehmen ist, der Anschluß an ein Elektrizitätswerk billiger als der Eigenbetrieb. Die Berliner Tarife allerdings machen beihöherenBetriebszeiten eine Ausnahme, und neben ihnen gibt es in Deutschland noch eine Anzahl Elektrizitätswerke, welche denselben Nachteil haben. Glücklicherweise nimmt ihre Anzahl aber immer mehr ab, und die Werke gelangen zu derEinsicht, daß es vorteilhafter für sie ist, ihre Großabnehmertarife mehr den Selbstkosten von Eigenanlagen anzupassen, mit denen sie schließlich doch konkurrieren müssen. Auch die Berliner Elektricitäts-Werke werden mit dieser Maßnahme nicht mehr lange warten lassen. In vielen Industriestädten sind jedoch die Strompreise so niedrig, wie in den hier angegebenen, und es ist zu berücksichtigen, daß die Tarife nur eine Grundlage für die Strompreise, nicht aber eine end- 
gültige Festsetzung bedeuten sollen. Es dürfte daher nicht zu viel behauptet sein, wenn der Satz aufgestellt wird: Fast jedes größere Elektrizitätswerk ist in der Lage, einer normalen Fabrik die Energie billiger zu liefern, als dieselbe sie erzeugen kann, und wird in seinem eigenen Interesse auch eine Verkaufspolitik in dieser Richtung treiben. Betrachtet man die Kurven im einzelnen genauer, so fällt noch folgendes auf:

Bei niedriger durchschnittlicher Belastung oder auch bei niedrigen Betriebszeiten stellt sich eine Eigenkraftanlage fast immer erheblich teurer als ein Anschluß an eine Zentrale. Selbst die Berliner Elektricitäts-Werke mit ihren ziemlich hohen Tarifen sind hier noch konkurrenzfähig. Im allgemeinen liegt die durchschnittliche Belastung einer Fabrik ungefähr zwischen 0,4 und 0,7. Auch in diesem Falle können die meisten Elektrizitätswerke Deutschlands mit den Eigenanlagen recht gut in Wettbewerb treten, wenigstens solange die Betriebszeiten nicht über das normale Maß, d. i. 3000 Std im Jahre, hinausgehen. Höhere Betriebszeiten sind selten; viele Großkraftwerke, in deren Versorgungsgebiet es solche Fabriken mit Nachtbetrieb gibt, haben ihren Tarif aber auch danach eingerichtet, so daß selbst bei 6000 Betriebsstunden und $3 / 4$ Belastung eine Reihe von Zentralen, wie aus den Schaubildern zu erkennen ist, noch billigere Energie liefern, als sie die Fabrik selbst sich herstellen könnte. In Beispiel 2 kommen die Kurven der Strombezugskosten denen des Eigenbetriebes ziemlich nahe. Dies liegt daran, daß hier keine Reservemaschine vorhanden ist. In vielen Fällen wird man aber der Betriebssicherheit halber eine solche aufstellen, wodurch sich der Vergleich zu ungunsten der Eigenanlage verschiebt.

Aus diesen Ausführungen gehen wohl ohne weiteres die Ursachen hervor, weshalb immer mehr Fabriken in Deutschland ihre eigenen Kraftanlagen aufgeben und sich an Elektrizitätswerke anschließen. Eine Ausnahme davon machen nur manchmal Fabriken, welche die Abwärme ihrer Kraftmaschinen verwerten können oder billige Abfallprodukte haben, die sich als Betriebsmittel für Kraftmaschinen eignen. In solchen Fällen ist die Eigenerzeugung der Energie sehr häufig billiger als deren Bezug von einerZentrale. Wenn trotzdem derartige Werke, selbst ganz große Industrieunternehmungen, dazu übergehen, ihren eigenen Kraftbetrieb zu verlassen und von einem Großkraftwerk Strom zu beziehen, so sind die mannigfachen Vorteile maßgebend, welche ihnen aus diesem Vorgehen erwachsen. Einmal spielt die Ersparnis an Anlagekapital eine große Rolle. Die auf einmal aufzubringenden Kosten für eine eigene Anlage sind recht groß, und das dafür aufzuwendende Kapital ist nicht immer vorhanden oder wird an einer anderen Stelle des Betriebes nötig gebraucht, wo es vielleicht auch besser ausgenutzt werden kann. Dann ist das Unternehmen für den Fall einer Vergrößerung bei Strombezug gar nicht behindert. Bei einer eigenen Anlage dagegen wäre man unter Umständen gezwungen, die alten Maschinen zu entfernen, und es müßten unter hohen Kosten neue, größere und modernere angeschafft werden. In schlechten Zeiten, in denen die Fabrik nur gering beschäftigt ist, werden die Betriebskosten durch die doch stets benötigten Abschreibungen und Zinsen für eine Eigenanlage stark beansprucht,

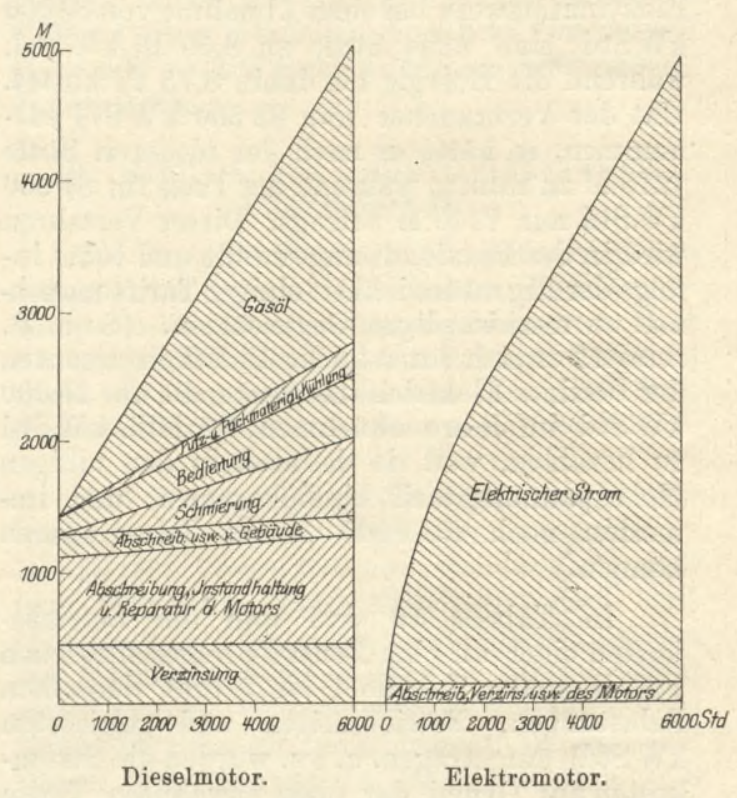

Abb. 3: Betriebskostenverglejch für einen Dieselmotor von $15 \mathrm{~kW}$ mit einem gleichstarken Elektromotor (Elektrizitätswerk Neuköllın) bei durchschnittlich $3 / 4$ Belastung.

während bei Strombezug ein großer Teil dieser Kosten wegfällt. Im umgekehrten Fall, bei sehr guter Konjunktur, in der ein Teil des Werkes mit Überstunden arbeiten muß, laufen während der Überstunden die Kraftmaschinen meist unterbelastet und mit schlechtem Wirkungsgrad. Bei Strombezug und Gruppen- bzw. Einzelantrieb ist dagegen kein Mehrverbrauch vorhanden. Außerdem ist die Betriebssicherheit in einer eigenen Anlage, besonders, wenn sie klein ist, nicht zu vergleichen mit der eines Elektrizitätswerkes. Störungen sind hier äußerst selten, in eigenen Kraftbetrieben, in denen noch dazu oft jedwelche Reserven fehlen, wie in unserem 


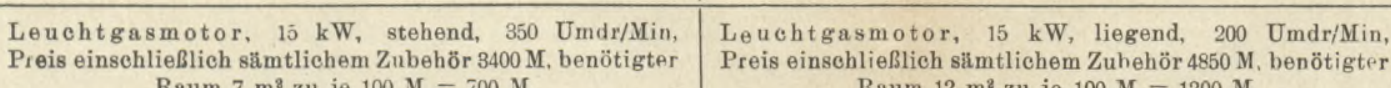

dauer von Stunden Von der Belastung unab.
hängige Kosten:

$5 \%$ Verzinsung der Masehinenkosten $\mathrm{M}$

des Motors …...... M

$5 \%$ Verzinsung, $2 \%$ Absehreibung.

$0,5 \%$ Instandhaltung $=8 \%$ der Ge-
băudekosten Sehmierölkosten

Bedienungskosten

Insgesamt von der Belastung unabhăngige
Kosten

Von der Belastung abhängige

bei durehsehnittlieh $1 / 4$ Belastung:

An der Motorwelle geleistete kWStd

Putz- und Packungsmaterialkosten
Kühlwasserkosten

Whiebsmittelkoste
Betriesten

Gesamtbetriebskosten pro Jahr

b) bei durehsehnittlieh $1 / 2$ Belastung:

An der Motorwelle geleistete kW Std $\ldots$

Putz. und Packungsmaterialkosten
Kühlwasserkosten

Betriebsmittelkoste

Gesamtbetriebskosten pro Jahr

e) bei durehsehnittlich $3 / 4$ Belastung.

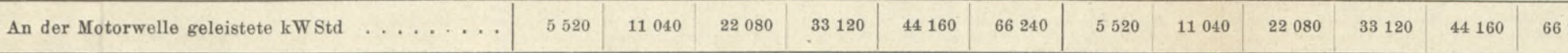

Putz- und Packungsmaterialkosten

Tühlwasserkosten

Gesamtbetriebskosten pro Jahr
Tabelle 2. Raum $7 \mathrm{~m}^{2}$

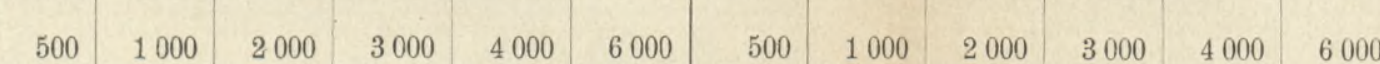

Tabelle 3.

Tabelle 4.

Tabelle 5.

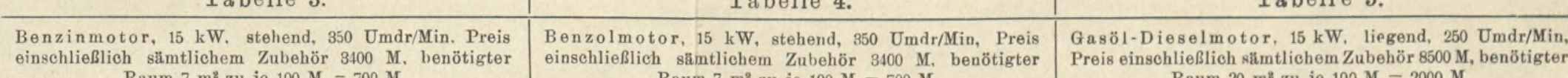
\begin{tabular}{|l|l|l|l|l|l|l|l|l|l|l|l|l|l|l|l|l|l|}
\hline 500 & 1000 & 2000 & 3000 & 4000 & 6000 & 500 & 1000 & 2000 & 3000 & 4000 & 6000 & 500 & 1000 & 2000 & 3000 & 4000 & 6000 \\
\hline
\end{tabular}

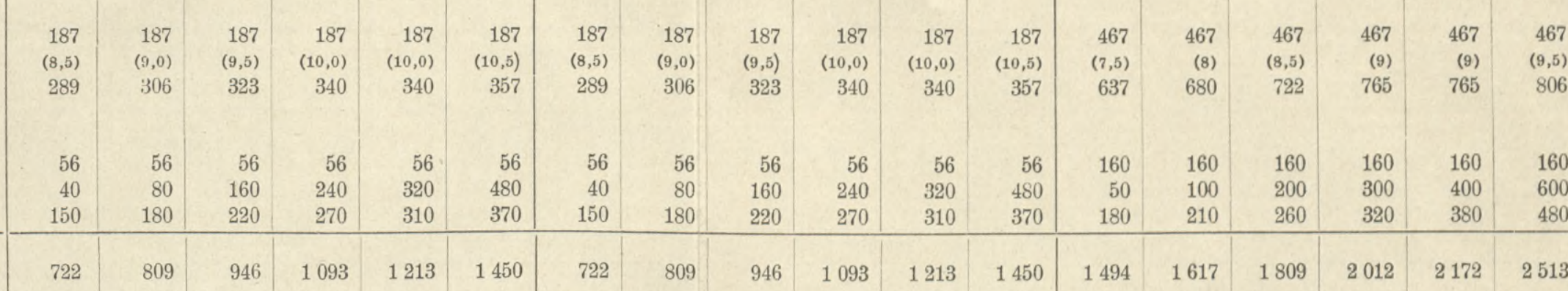

\begin{tabular}{|l|l|l|l|l|l|l|l|l|l|l|l|}
\hline 722 & 809 & 946 & 1093 & 1213 & 1450 & 917 & 1011 & 1156 & 1310 & 1454 & 167 \\
\hline
\end{tabular}

180

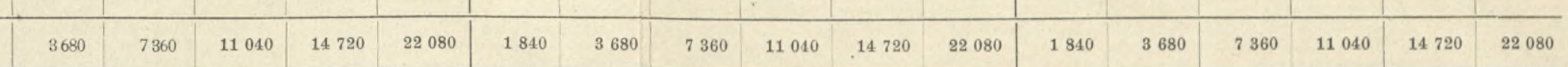

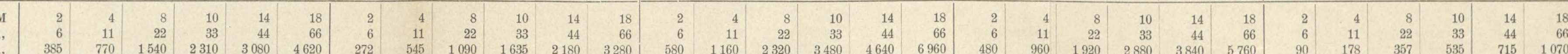

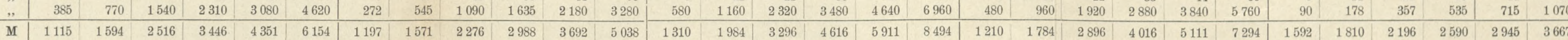

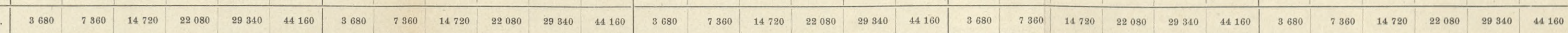

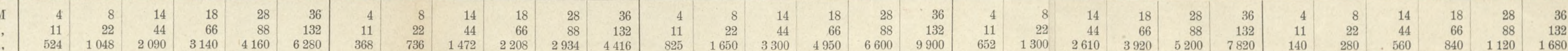

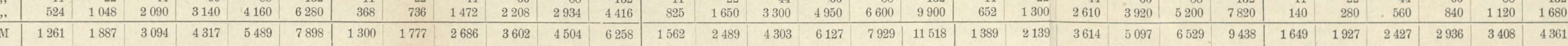

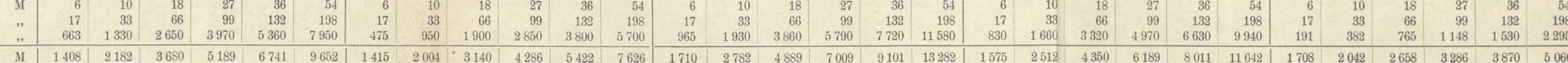

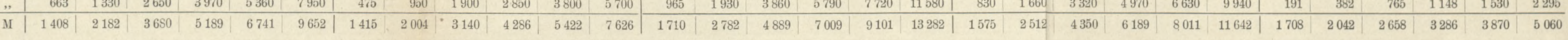


Tabelle 6.

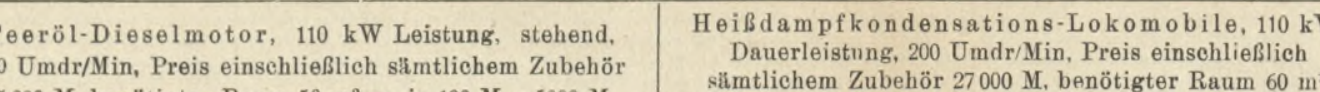
$45000 \mathrm{M}$, benôttigter Raum $50 \mathrm{~m}^{2}$ zu je $100 \mathrm{M}=5000 \mathrm{M}$.

etriebskosten bei einer jährlichen $\mathrm{Be}$ \begin{tabular}{l|l|l|l|l|l|}
500 & 1000 & 2000 & 3000 & 4000 & 6000
\end{tabular}

Von der Belastung unabhängige Kosten:
ung der Maschinenkosten
und Instandhaltung ders Kraftmasehine. Instandhaltung $5,5 \%$ Verzinsung, $2 \%$ Absehreibung,
$1 / 2 \%$ Instandhaltung $=8 \%$ der Ge-
bäudekosten. schmierölkoste Bedienungskosten

Kosten für Anheize

Kühlwasserkost
Zündölkosten .

Insgesamt von der Belastung unabhängigo Kosten ..............

Von der Belastung abhängigo

2) Bei durehsehnittlich $1 / 4$ Belastung:

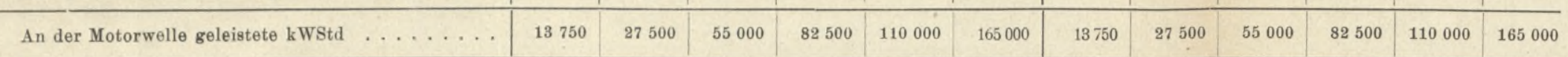

Putz-und Packungsmaterialkosten

Kühlwasserkosten
Betriebsmittelkosten

esamtbetriebskosten pro Jahr

\begin{tabular}{|l|r|r|r|r|r|r|r|r|r|r|r|r|}
\hline M & 8 & 15 & 30 & 45 & 60 & 90 & 8 & 15 & 30 & 45 & 60 & 90 \\
\hline & 29 & 58 & 115 & 173 & 230 & 345 & - & - & - & - & - & -9 \\
\hline
\end{tabular}

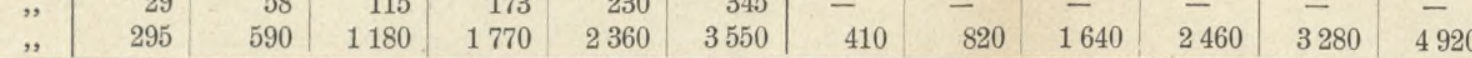
b) Bei durehsehnittlich $1 \frac{1}{2}$ Belastung:

$$
\text { An der Motorwelle geleistete } \mathrm{kWStd} \text {. ... }
$$

\begin{tabular}{|l|l|l|l|l|l|l|l|l|l|l|l||}
7297 & 8458 & 10265 & 11883 & 13270 & 16275 & 5578 & 6885 & 9150 & 11285 & 13180 & 17110 \\
\hline
\end{tabular}

Putz- und Packungsmaterialkosten Betriebsmittelkosten

Gesamtbetriebskosten pro Jahr

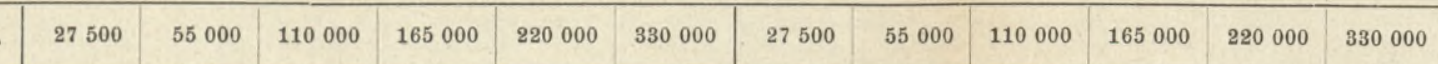

Bei durehsehnittlich $3 / 4$ Belastung An der Motorwelle geleistete kWStd

\begin{tabular}{|l|r|r|r|r|r|r|r|r|r|r|r|r|}
\hline M & 15 & 30 & 60 & 90 & 120 & 180 & 15 & 30 & 60 & 90 & 120 & 180 \\
,$"$ & 58 & 115 & 230 & 345 & 460 & 790 & - & - & - & - & - & - \\
\hline
\end{tabular}

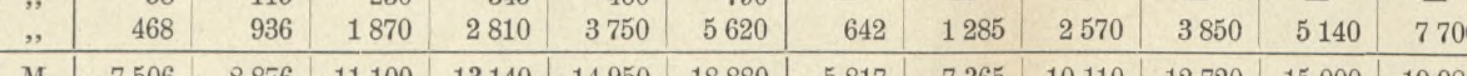
Putz- und Packungsmaterialkosten Betriebsmittelkosten

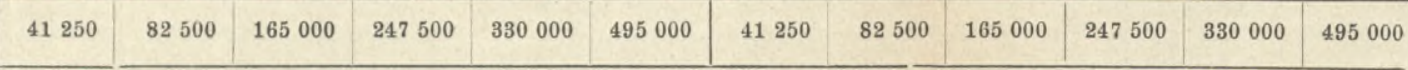
Gesamtbetriebskosten pro Jahr \begin{tabular}{|r|rr|r|r|r|r|r|r|r|r|r|r|}
\hline M & 23 & 45 & 90 & 135 & 180 & 270 & 23 & 45 & 90 & 135 & 180 & 27 \\
\hline & 87 & 174 & 345 & 520 & 790 & 1040 & - & - & - & - & - & \\
\hline
\end{tabular} \begin{tabular}{|r|r|r|r|r|r|r|r|r|r|r|r|r|}
\hline, & 620 & 1240 & 2480 & 3720 & 4960 & 7440 & 870 & $\overline{1740}$ & -3480 & $\overline{5} 220$ & $\overline{6960}$ & $\overline{10440}$ \\
\hline
\end{tabular}
Tabelle 8.

Tabelle 9 .

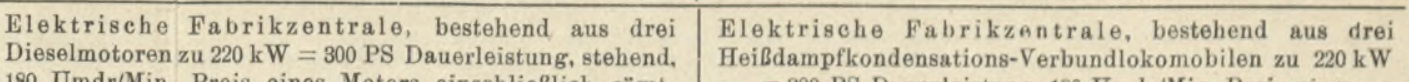
lichem Zubehör 80000 M. und drei Gleichstrom generatoren zun je $200 \mathrm{~kW}$,

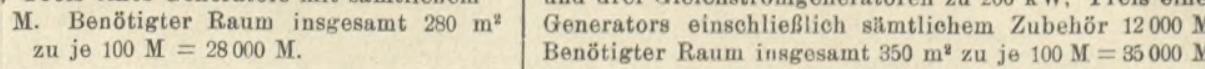

Betriebskosten bei einer jährlichen Betriebsdauer von Stunden

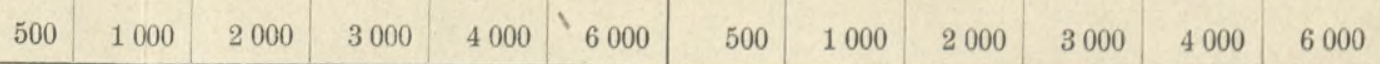
Von der Belastung unabhängige
Kosten: $5,5 \%$ Verzinsung der gesamten Ma sehinenanlage der Kraftmaschinen A bsohreibung und Instandhaltung der Generatoren $0.5 \%$ Instandhaltung $=8 \%$ der $\mathrm{G}$ bäudekosten
Schmierölkosten Bedienungskosten . . Kosten für das An
Kühlwasserkosten

ndölkosten .

Insgesamt von der Belastung unabhăngige
Kosten

Von der Belastung abhängige

a) Bei durehsehnittlich $1 / 4$ Belastung:

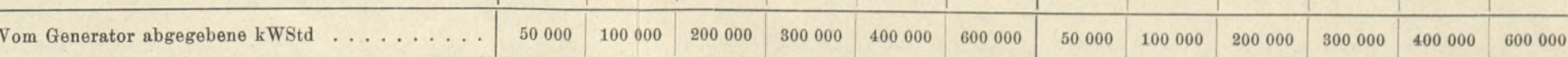
\begin{tabular}{|l|r|r|r|r|r|r|r|r|r|r|r|r|r|}
\hline $\begin{array}{l}\text { Putz- und Packungsmaterialkosten } \\
\text { Kint }\end{array}$ & M & 20 & 40 & 80 & 120 & 160 & 240 & 20 & 40 & 80 & 120 & 160 & 240 \\
\hline
\end{tabular} Kühlwasserkosten Gesamtbetriebskosten pro Jal \begin{tabular}{|r|r|rrrrrrrrr|r|r|r|}
\hline, & 1260 & 2525 & 5050 & 7575 & 10100 & 15150 & $\overline{1520}$ & $\overline{3} 040$ & $\overline{6} 080$ & $\overline{9120}$ & 12160 & 18240 \\
\hline
\end{tabular} \begin{tabular}{l|l|l|l|l|l|l|l|l|l|l|l|l}
. M & 40485 & 44275 & 50626 & 56995 & 62180 & 73530 & 28930 & 33380 & 40050 & 46030 & 51220 & 62480
\end{tabular}

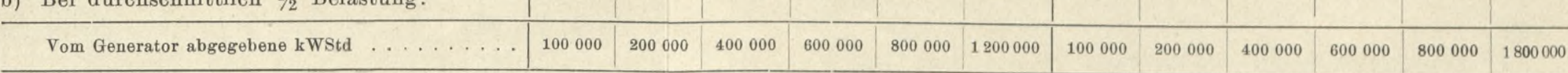
\begin{tabular}{|l|r|r|r|r|r|r|rrrrr|r|r|r|}
\hline Putz- und Packungsmaterialkosten - M & 40 & 80 & 160 & 240 & 320 & 480 & 40 & 80 & 160 & 240 & 320 & 480 \\
\hline
\end{tabular}

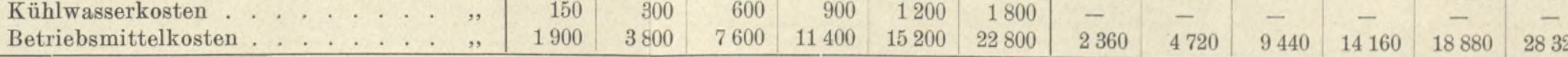
\begin{tabular}{|l|l|l|l|l|l|l|l|l|l|l|l|l|l}
\hline Gesamtbetriebskosten pro Jahr . . . . . . & M & 41220 & 45740 & 53550 & 61390 & 68040 & 82320 & 29790 & 35100 & 43490 & 51190 & 58100 & 72800
\end{tabular} c) Bei durehsehnittlich $3 / 4$ Belastung:

Vom Generator abgegebene kWSt

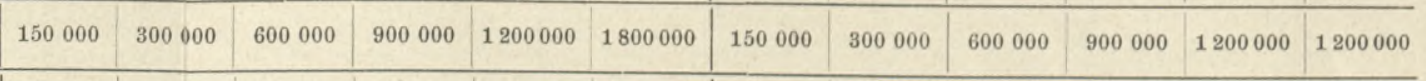
\begin{tabular}{|lr|r|r|r|r|r|r|r|r|r|r|r|r|}
\hline Putz- und Packungsmaterialkosten & M & 60 & 120 & 240 & 360 & 480 & 720 & 60 & 120 & 240 & 360 & 480 & 720 \\
\hline
\end{tabular}

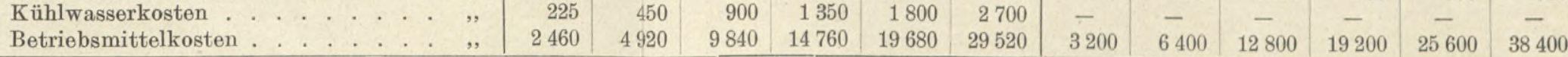

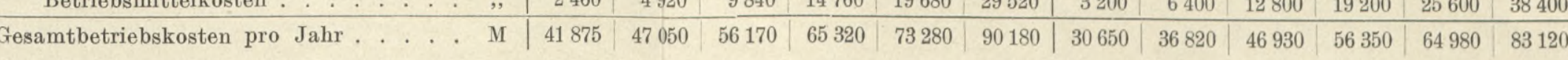



Tabelle 10 .

\begin{tabular}{|c|c|c|c|c|c|c|}
\hline \multirow[b]{2}{*}{$\begin{array}{l}\text { Betriebskosten bei einer jährlichen Be- } \\
\text { triebsdauer von Stunden } . . . .\end{array}$} & \multicolumn{6}{|c|}{$\begin{array}{l}\text { Drehstrommotor, } 15 \mathrm{~kW} \text { Leistung, } 1500 \mathrm{Umdr} / \mathrm{Min}, \\
\text { Preis einsehließlich sümtlichem Zubehör } 1050 \mathrm{M} \text {. Benötigter } \\
\text { Raum } 2 \mathrm{~m}^{2} \mathrm{zu} \text { je } 100 \mathrm{M}=200 \mathrm{M} \text {. }\end{array}$} \\
\hline & 500 & 1000 & 2000 & 3000 & 4000 & 6000 \\
\hline 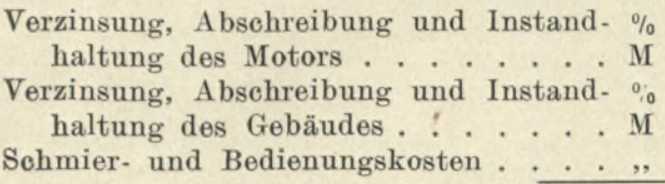 & $\begin{array}{r}(12,0) \\
126 \\
(8) \\
16 \\
10\end{array}$ & $\begin{array}{r}(12) \\
126 \\
(8) \\
16 \\
10\end{array}$ & $\begin{array}{r}(12,2) \\
128 \\
(8) \\
16 \\
10\end{array}$ & $\begin{array}{r}(12,2) \\
128 \\
(8) \\
16 \\
10\end{array}$ & $\begin{array}{r}(12,5) \\
131 \\
(8) \\
16 \\
10\end{array}$ & $\begin{array}{r}(12,5) \\
131 \\
(8) \\
16 \\
10\end{array}$ \\
\hline $\begin{array}{l}\text { Insgesamt von der Belastung unabhängige } \\
\text { Kosten. } \\
\text { Von der Belastung abhängige } \\
\text { Kosten: } \\
\text { a) Bei durehsehnittlich } 1 / 4 \text { Belastung: }\end{array}$ & 152 & 152 & 154 & 154 & 157 & 157 \\
\hline
\end{tabular}

\begin{tabular}{|c|c|c|c|c|c|c|}
\hline An der Motorwelle geleistete $\mathrm{kWStd} \ldots \ldots$ & 1840 & 3680 & 7360 & 11040 & 14720 & 22080 \\
\hline
\end{tabular}

Städtisches Elektrizitätswerk Neukölln:

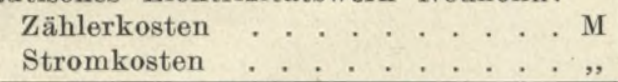
\begin{tabular}{|r|r|r|r|r|r|r|}
\hline & 24 & 24 & 24 & 24 & 24 & 120 \\
\hline & 302 & 604 & 1208 & 1812 & 2416 & 2880 \\
\hline
\end{tabular} \begin{tabular}{|l|l|l|l|l|l|l|l|}
\hline Gesamtbetriebskosten pro Jahr . . . M & 478 & 780 & 1386 & 1990 & 2597 & 3157 \\
\hline
\end{tabular}

Berliner Vororte: Drehstromnetz oberirdisch: \begin{tabular}{|r|r|r|r|r|r|r|r}
\hline Stromkosten ......... . M & 232 & 464 & 928 & 1392 & 1856 & 2784 \\
\hline Gesamtbetriebskosten pro Jahr .... M & 384 & 616 & 1082 & 1546 & 2013 & 2941
\end{tabular}

Rheinisch-Westfälisches Elektrizitätswerk: Zählerkosten ........... M Stromkosten

M

\begin{tabular}{r|r|r|r|r|r|r}
24 & 24 & 24 & 24 & 24 & 24 \\
\hline
\end{tabular} \begin{tabular}{|l|l|l|l|l|l|l|l|}
\hline Gesamtbetriebskosten pro Jahr .... M & 501 & 826 & 1338 & 1718 & 2031 & 2601
\end{tabular}

b) Bei durehschnittlich $1 / 2$ Belastung:

\begin{tabular}{|c|c|c|}
\hline \multicolumn{3}{|c|}{ An der Motorwelle geleistete $\mathrm{kWStd} \ldots \ldots$} \\
\hline Städtisches Elekt & zitätswerk & Neukölln: \\
\hline $\begin{array}{l}\text { Zählerkosten } \\
\text { Stromkosten }\end{array}$ & $\begin{array}{lll}. & . & . \\
. & . & .\end{array}$ & $\begin{array}{lll}. & . & \mathrm{M} \\
. & . & \end{array}$ \\
\hline
\end{tabular}

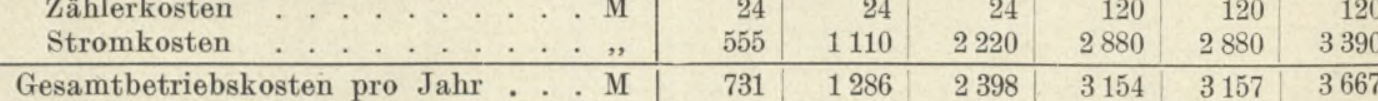

Städtisches Elektrizitätswerk Dortmund:

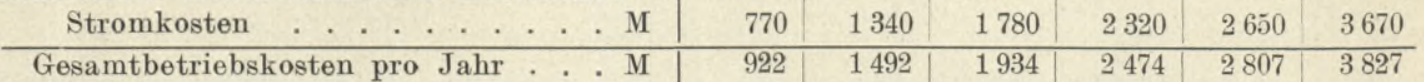

Rheinisch-Westfälisches Elektrizitätswerk: Zählerkosten \begin{tabular}{|l|r|r|r|r|r|r}
\hline & 24 & 24 & 24 & 24 & 24 & 24 \\
\hline
\end{tabular} \begin{tabular}{|l|l|l|l|l|l|l|}
\hline Gesamtbetriebskosten pro Jahr $\ldots . . M$ & 773 & 1236 & 1948 & 2468 & 2891 & 3731
\end{tabular}

c) Bei durehschnittlich $3 / 4$. Belastung:

\begin{tabular}{|c|c|c|c|c|c|c|}
\hline An der Motorwelle geleistete $\mathrm{kW} \mathrm{Std}$ & 5520 & 11040 & 22080 & 33120 & 44160 & 66240 \\
\hline Städtisohes Elektrizitätswerk Neukölln: & & & & & & \\
\hline Zählerkosten . . . . . . M & 24 & 24 & 120 & 120 & 120 & 120 \\
\hline Stromkosten & 820 & 1640 & 2880 & 2960 & 3360 & 4030 \\
\hline Gesamtbetriebskosten pro Jahr ... M & 996 & 1816 & 3154 & 3234 & 3637 & 4307 \\
\hline $\begin{array}{l}\text { Städtisehes Elektrizitätswerk Dortmund: } \\
\text { Stromkosten } \\
\text {. . . . . . . M }\end{array}$ & 1103 & 1350 & 2160 & 2860 & 3520 & 4780 \\
\hline Gesamtbetriebskosten pro Jahr ... M & 1255 & 1502 & 2314 & 3014 & 3677 & 4937 \\
\hline Rheinisch-Westfälisches Elektrizitätswer & & & & & & \\
\hline$\cdots \cdots \mathrm{M}$ & 24 & 24 & 24 & 24 & 24 & 24 \\
\hline Stromkosten . . . . . . . , & 820 & 1400 & 2240 & 2860 & 3500 & 4770 \\
\hline Gesamtbetriebskosten pro Jahr & 996 & 1576 & 2418 & 3038 & 3681 & 4951 \\
\hline
\end{tabular}

Tabelle 11.

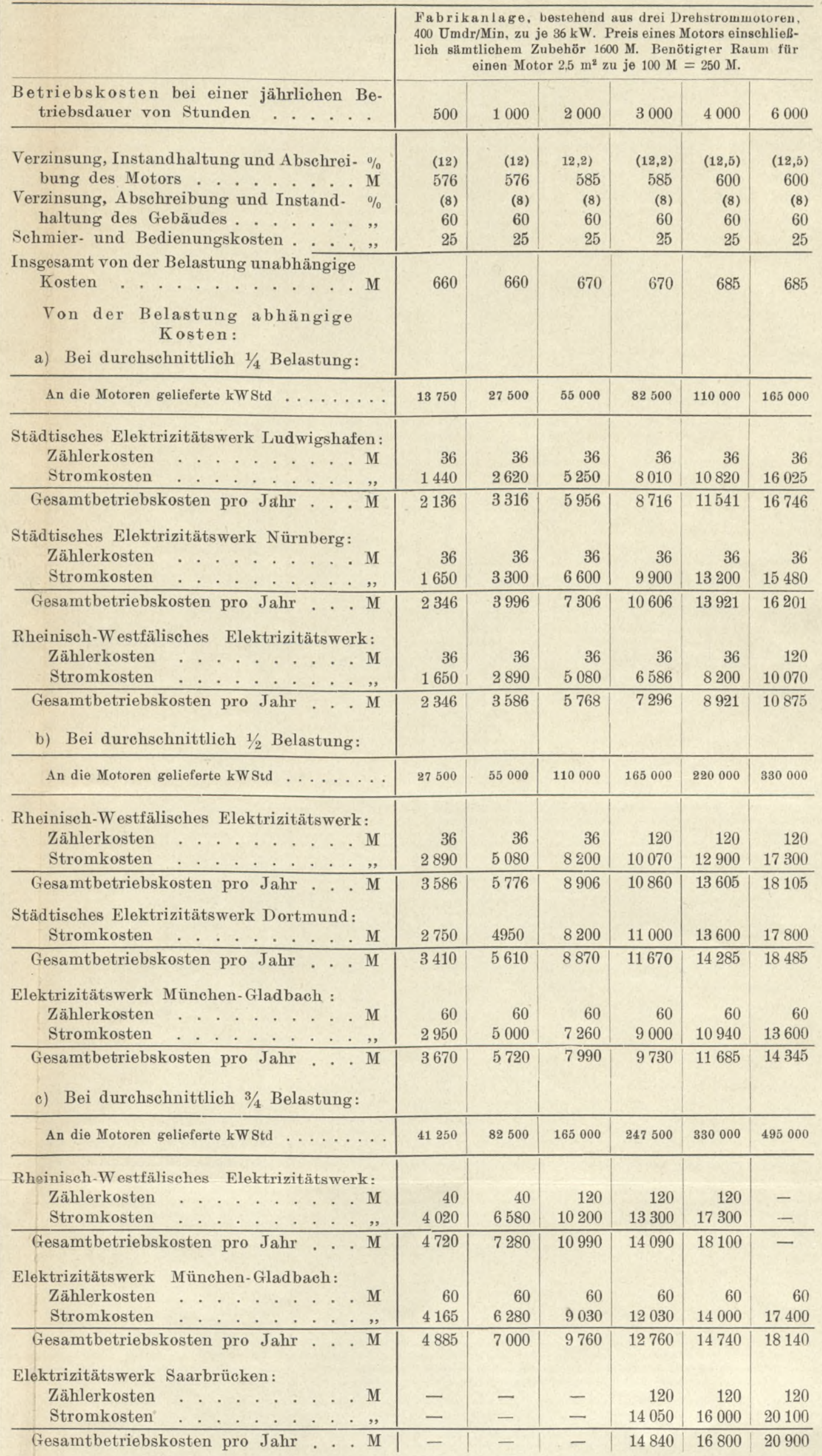




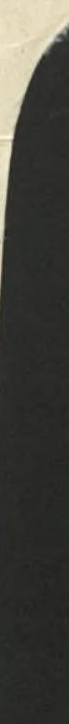




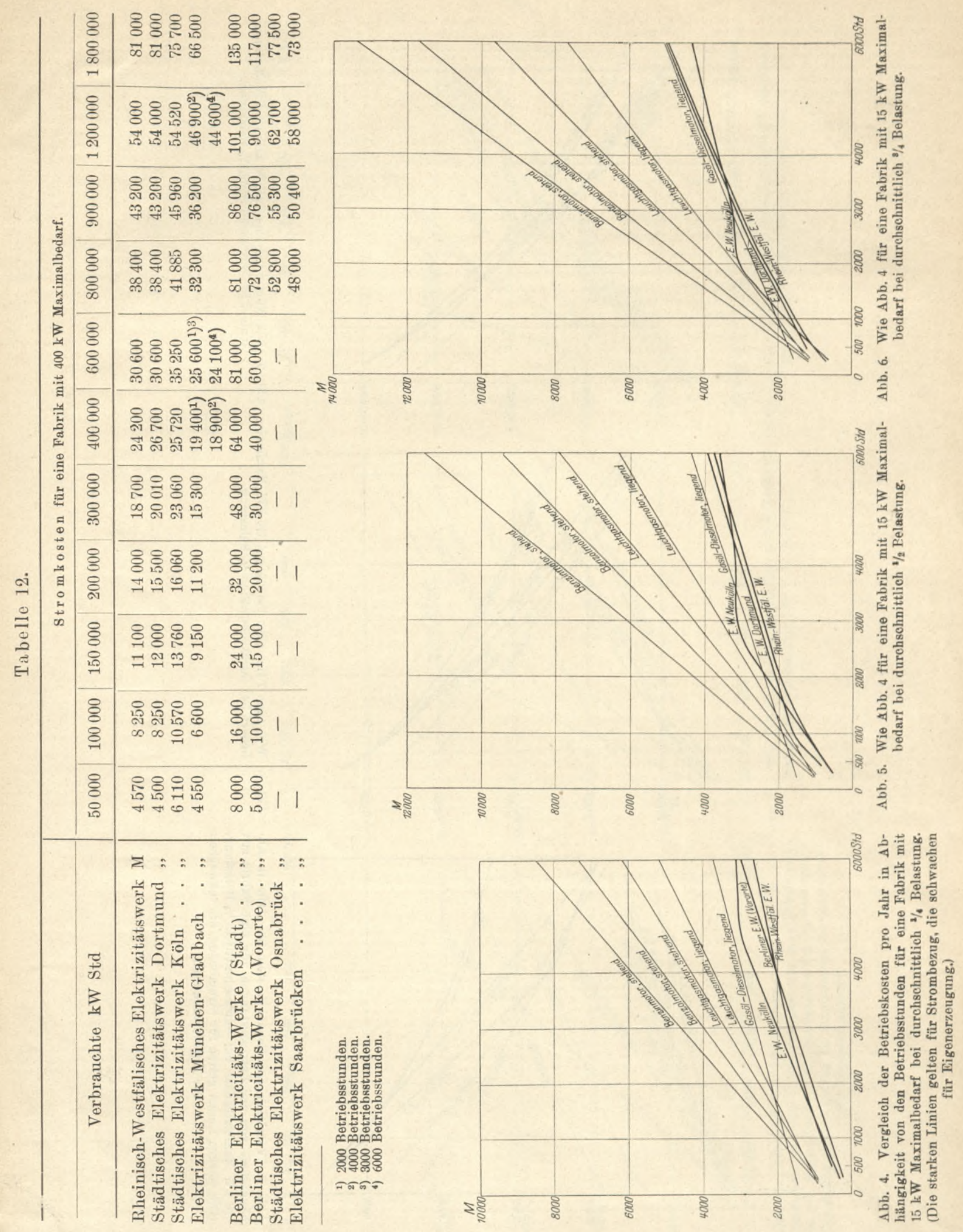



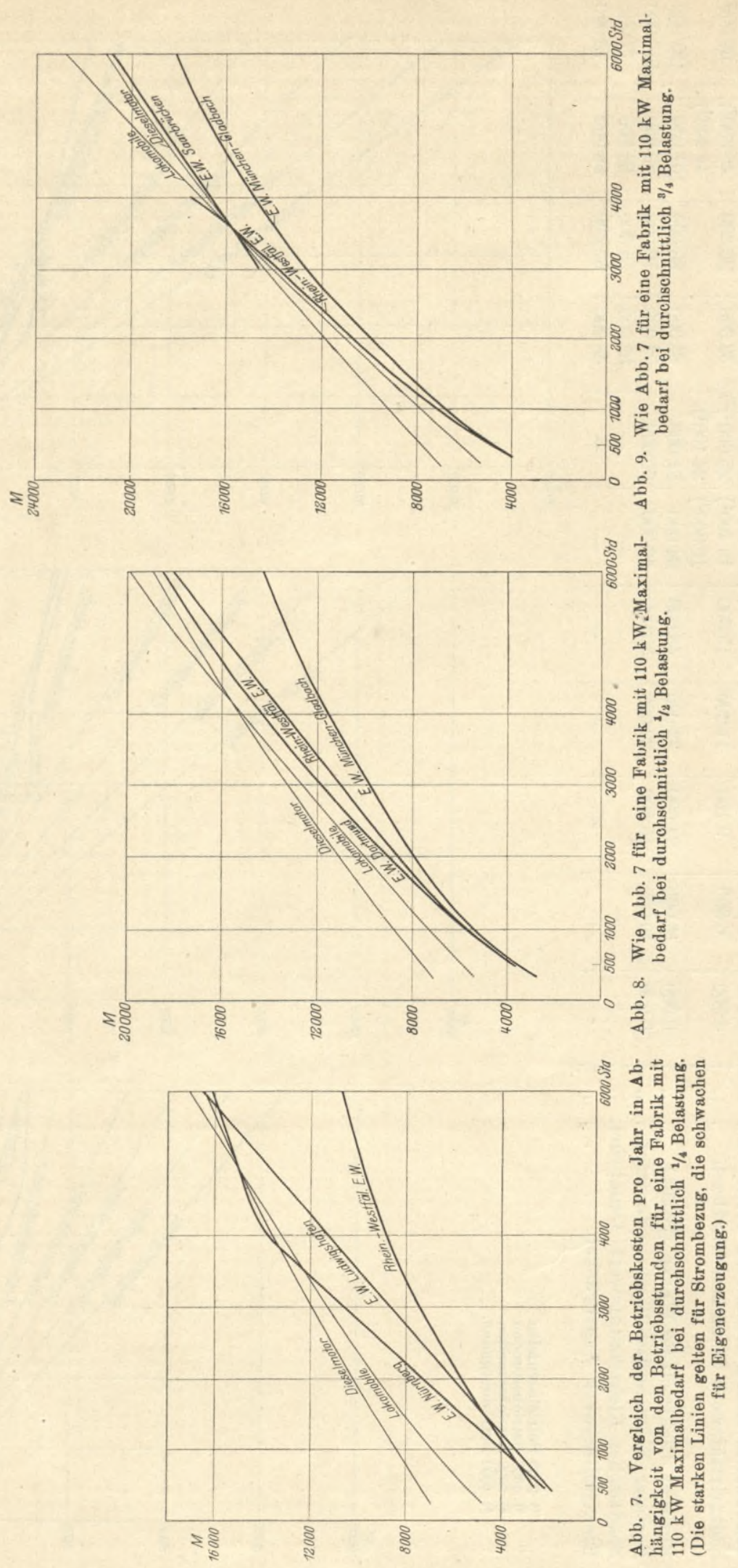


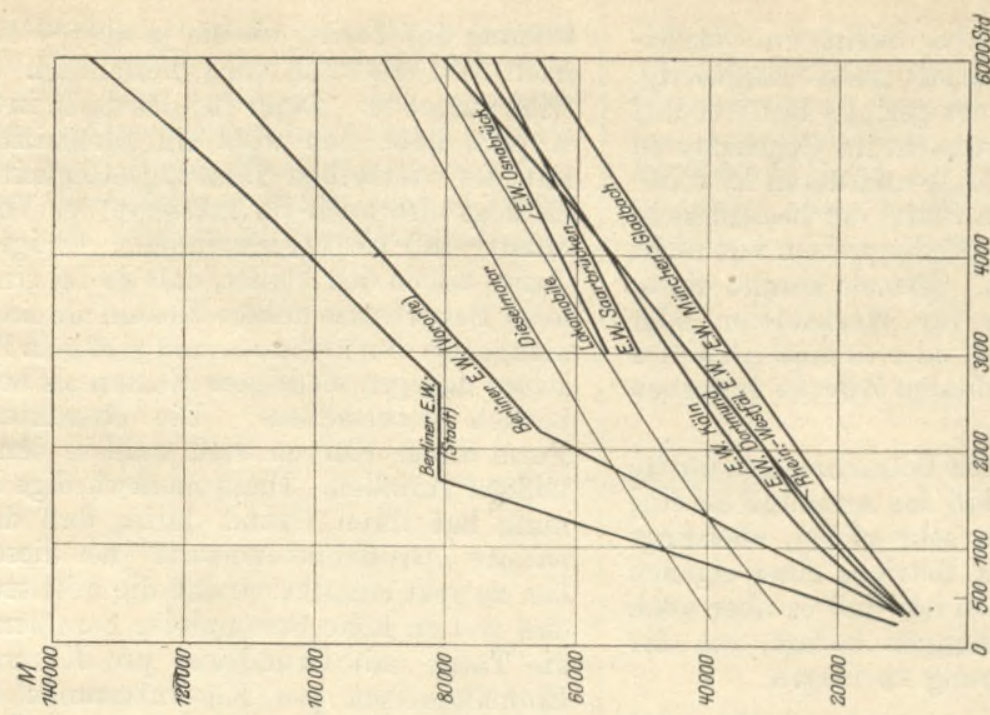

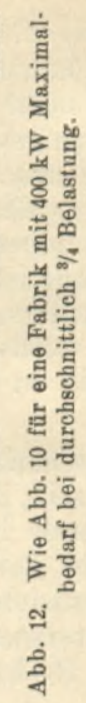
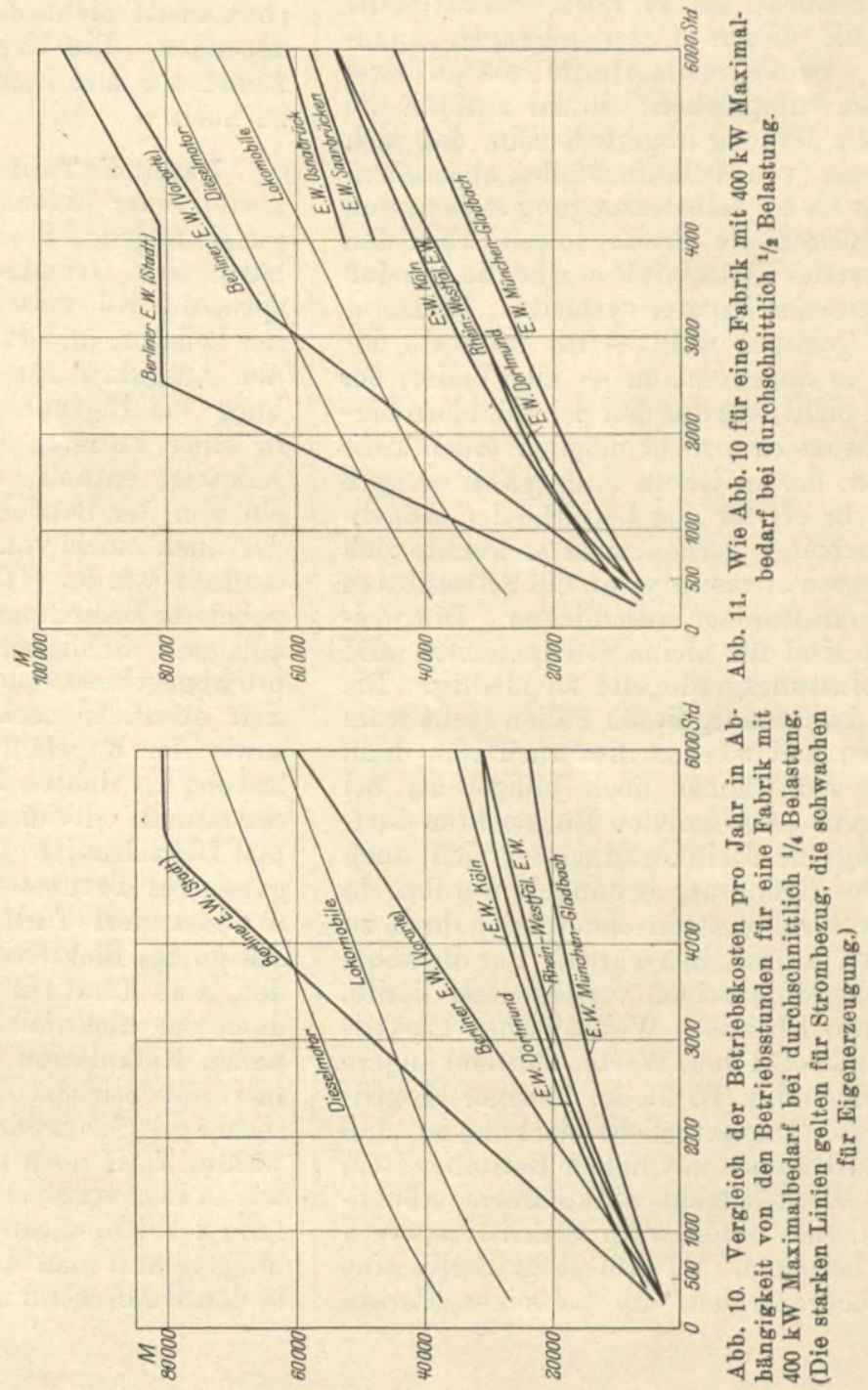
Rechnungsbeispiel 2, aber nicht zu vermeiden und dann manchmal recht langwierig. Schließlich wäre noch der geringe Raumbedarf bei Strombezug zu erwähnen, im Gegensatz zu dem für eine Eigenzentrale und deren Erweiterung benötigten großen Platz, der besonders in Großstädten mit hohen Bodenpreisen von nicht geringer Bedeutung ist. Oftmals könnte dieser Raum wirtschaftlicher für Werkstätten oder Lager verwandt werden, oder es muß ein teures Grundstück allein zu diesem Zwecke erworben werden.

Aus den vorstehenden Betrachtungen dürfte hervorgegangen sein, daß der Anschluß an eine Elektrizitätszentrale in sehr vielen, allerdings nicht allen Fällen dem Betriebe einer eigenen Kraftanlage vorzuziehen ist, daß es aber auch eingehender Untersuchungen bedarf, um der jeweiligen Lage Rechnung zu tragen.

Zum Schlusse sei es noch erlaubt, eine kurze Kritik diesen Untersuchungen anzuschließen. Großabnehmertarife sollten zwei Forderungen entsprechen: einmal müßten die Stromkosten derartig abgestuft sein, daß sich die Abnehmer (von Ausnahmefällen abgesehen) nicht teurer als bei Selbsterzeugung stehen, und zweitens müssen die Preise soweit über den Selbstkosten des Elektrizitätswerkes liegen, daß ein angemessener Nutzen verbleibt. Letzterer Forderung genügen wohl - im Interesse der Werke sei es angenommen - alle Tarife, der ersten aber nicht, wie aus den Schaubildern hervorgeht. Es ist zwar nicht möglich, einen Tarif aufzustellen, der in jedem Falle paßt, er muß vielmehr sehr oft für den betreffenden Abnehmer umgearbeitet werden, aber er könnte sich zum mindesten etwas enger an die Selbstkosten einer Eigenkraftanlage anschließen. Die meisten Tarife sind für kleine Betriebszeiten und geringe Belastungsgrade viel zu niedrig. Die Abnehmer könnten in diesen Fällen recht wohl mehr zahlen und würden dies auch tun, denn sie stehen sich immer noch billiger als bei Eigenanlagen. (Aus sozialen Rücksichten dürften allerdings die Kleinkonsumenten nicht auch nach diesem Grundsatz behandelt werden, da sonst der wertvolle motorische Antrieb ihnen zu hohe Kosten verursachen würde.) Für die höheren Betriebszeiten zeigen verschiedene Tarife, z. B. der des Rheinisch-Westfälischen Elektrizitätswerkes, recht gute Werte, während andere viel zu hoch sind, z. B. die der Berliner Elektricitäts-Werke. Die natürliche Wirkung ist, daß viele Großabnehmer mit hohen Betriebszeiten, die ja den Werken recht willkommene Abnehmer wären, sich hüten, vom Elektrizitätswerk Strom zu beziehen. So gibt es in Berlin eine Menge Blockzentralen, die bei einer Herab- setzung des Tarifs, wie sie ja aller Voraussicht nach bald eintreten wird, hoffentlich den Betrieb aufgeben. Denn für das darin investierte Kapital ließe sich wohl ein Elektrizitätswerk mit der vierfachen Leistungsfähigkeit bauen. Es liegt also auch im Interesse der Volkswirtschaft, daß sie verschwinden. Einige andere Tarife haben den Fehler, daß sie bei einer mittleren Betriebszeit höhere Kosten als eine Eigenanlage, bei den kleineren und größeren Betriebszeiten dagegen niedrigere Kosten als bei Eigenbetrieb verursachen. Die charakteristische Form dieser Kurven wird wohl in den Schaubildern auffallen. Diese merkwürdige Erscheinung hat ihren Grund darin, daß der sogenannte „Großabnehmertarif" bei diesen Werken zu spät einsetzt, so daß die mittleren Kunden viel $\mathrm{zu}$ hohe Strompreise bezahlen. Auch die Tarife mit Grundtaxe pro Kilowatt und Einheitspreisen pro Kilowattstunde entsprechen meist nicht den oben aufgestellten Forderungen. Die Grundgebühr ist für kleinere Abnehmer fast immer zu niedrig, für größere zu hoch.

Damit die Tarife sich vollständig den Selbst. kosten einer Eigenkraftanlage anpassen, müßten sie folgende Form besitzen: der Abnehmer hätte eine Grundgebühr pro maximal beanspruchtes Kilowatt zu zahlen, die sich je nach der Leistung ändert. (Diese Gebühr entspricht den Ausgaben für Verzinsung und Abschreibung bei Eigenanlagen. Sie ließe sich evtl. in einer Formel, die irgend eine Potenz der Kilowatt enthält, ausdrücken.) Dazu käme ein von der Betriebszeit abhängiger Zuschlag, der auch noch von der Maximalleistung beeinflußt würde. (Dieser entspricht den Ausgaben für Bedienung und Schmierung bei Eigenanlagen.) Schließlich wäre noch ein Zuschlag pro abgegebene Kilowattstunde zu zahlen, der sich ebenfalls nach der Höhe der Leistung sowie der ungefähren durchschnittlichen Belastung zu richten hätte (= abgegebene Kilowattstunde dividiert durch Maximalleistung mal Betriebszeit). (Dieser entspricht den Ausgaben für Brennstoff bei Eigenanlagen.) Der so konstruierte Tarif müßte nun noch den Selbstkosten des Elektrizitätswerkes angenähert werden, was durchaus nicht schwierig sein wird, denn ein Elektrizitätswerk muß bei einer genauen Kalkulation für jeden einzelnen Abnehmer mit beinahe denselben Kostenelementen rechnen. Schließlich müßte der wirklich gewählte Tarif noch etwas einfacher sein, denn, wie es hier vorgeschlagen ist, wäre die Ermittlung der Stromkosten recht kompliziert. Allerdings nimmt man eine derartige Rechnung gern in den Kauf, wenn man weiß, daß damit beiden 
Teilen gedient ist, und es verdient ja hiermit nicht nur der Besitzer des Elektrizitätswerkes, sondern auch der Abnęhmer, der gegenüber einer Eigenanlage eine größere Summe erspart. Natürlich wird dieser Tarif für verschiedene Orte ganz verschieden ausfallen, aber es dürfte wohl für jedes größere Elektrizitätswerk mög- lich sein, den Tarif so $\mathrm{zu}$ bemessen, daß fast alle Einzelanlagen aus dem Versorgungsgebiete verschwinden. Dies zu erreichen, ist ja eines der erstrebenswerten Ziele unserer großen Kraftwerke, und es ist $\mathrm{zu}$ hoffen, daß sie in nicht allzu ferner Zeit dahin gelangen werden. 


WYDZIAEY POLITECHNICZNE KRAKÓW

\section{BIBLIOTEKA GŁÓWNA}

IIII 33124

Kdn., Czapskich 4 - 678. 1. XII. 52. 10.000 